\title{
Exploration of the Federal Communications Commission's Experimental Radio Service (ERS): Understanding ten years of experimental spectrum licenses
}

\author{
Pedro Bustamante \\ (Corresponding Author) \\ School of Information \\ and Computing \\ University of Pittsburgh \\ Pittsburgh, PA \\ Email:pjb63@pitt.edu
}

\author{
Martin Weiss \\ School of Information \\ and Computing \\ University of Pittsburgh \\ Pittsburgh, PA \\ Email: mbw@pitt.edu
}

\author{
Douglas Sicker \\ Department of Engineering \\ and Public Policy \\ Carnegie Mellon University \\ Pittsburgh, PA \\ Email: sicker@cmu.edu
}

\begin{abstract}
The Experimental Radio Service (ERS) of the Federal Communications Commission (FCC) has awarded experimental licenses for more than thirty years as a means to promote research and innovation in wireless technologies. In this work, we present an exploratory analysis of the details pertaining to the assignment of these licenses during the past ten years (20072016). For this purpose, we have built a single repository of technical and non-technical details about license applications by scraping publicly available information in the FCC's website. This has permitted us systematically categorize among the existing types of experimental licenses and, subsequently, analyze their characteristics. We pay particular attention to the evolution of various parameters such as duration of license, frequency of assignment, processing times, operational parameters, among others. In addition, we explore potential trends hidden in ten years of experimental licenses. This allow us to better understand the time burden of obtaining an authorization or the factors that may influence the license granting process. We conclude this work by delving into the details behind the relationship between ERS authorizations and well known wireless technologies, in particular TV White Spaces and 5G.
\end{abstract}

\section{INTRODUCTION}

For innovation to be successful, it is necessary for the adequate resources to be readily available. Commonly, resource availability results from significant technical, regulatory and scientific investments. Regulatory bodies, like the Federal Communications Commission ${ }^{1}$, have made significant efforts in order to promote this innovation through its Experimental Radio Service.

The objective of the Commission's experimental licensing program is precisely to provide resources to different types of users, while promoting innovation. When a licensing system is implemented, it is interesting to observe its impact. The influence of commercial licenses is quite evident. Indeed, we can pay attention to multiple indicators that point to how

\footnotetext{
${ }^{1}$ Also referred as the "Commission" throughout different sections of this work.
}

valuable the auctioned spectrum is, and which are the uses that merit higher investments. In other cases, such as that of the ERS, those indicators are not that evident.

There is a significant amount of public information regarding these licenses; however, it is difficult to access the full data, and a deeper analysis is required to draw meaningful conclusions. Such an analysis can provide us with metrics to evaluate how onerous the process can be to obtain a license, but more importantly, it can allow us to find out whether and how an experimental licensing system has contributed to the development and improvement of the myriad technologies that have arisen. It is for this reason that in this work we tackle the task of delving deeper into the FCC's experimental licensing process.

Our first task is to obtain, organize and process all relevant information, taking into account metrics that we consider appropriate for our evaluation. Hence, we build a general experimental license database by scraping information from the FCC's website under the "Generic Search Platform". Having all the information in a single repository ${ }^{2}$, allows us to determine which parameters can be studied in more detail, including technical and non-technical details of not only the licenses themselves, but also the applicants seeking to obtain an experimental authorization. Ultimately, these parameters permit us to explore the applicability of experimental licenses in technologies such as the utilization of TV White Spaces or the development of the Fifth Generation of Cellular Networks $(5 \mathrm{G})$.

\section{Motivation}

The fast evolution of wireless technologies is undeniable. Nowadays, the proportion of users that have LTE service

${ }^{2}$ The FCC allows access to all non-confidential information about the ERS. Nevertheless, this information is contained in multiple repositories and designed for individual searches rather than bulk queries. 
in countries such as South Korea, United States or Japan is greater than $81 \%$ [20]. Furthermore, several efforts are being done to start deploying 5G by the first quarter of 2018 [22]. A critical part of these efforts is securing access to the spectrum bands where the technology will be deployed, as this represents one of the most innovative characteristics of 5G. Initial experiments have proposed the use of large chunks of underutilized spectrum in very high frequencies such as millimeter-wave (mmWave) [12], [21], [23]. For this idea to become part of a future standard, several deployments and tests in the field need to be carried out. It is here where experimental licenses can play a very important role. In fact, as discussed in [5], [16], [17], [26], ERS licenses are already being used for developing these new wireless technologies.

Nonetheless, we consider that a key factor for understanding the relationship between experimental licenses and future wireless technologies is to first study their basic features. To the best of our knowledge, no analysis has been done to explore the fundamental characteristics of the Experimental Radio Service of the FCC. This requires, first, finding a method for extracting, aggregating and processing the information that the FCC has made publicly available ${ }^{3}$. This allows us to perform a deeper analysis on relevant metrics and parameters about the usage of this type of license. We expect such an analysis to represent a useful tool for current and future exploratory activities around the relationship between the ERS and the development of new technologies, its policy considerations and its regulation implications.

\section{BACKGROUND}

\section{A. What are the experimental licenses?}

The FCC is authorized to provide experimental use of frequencies as amended in the Section 303(g) of the Communications Act of $1934^{4}$. This amendment assigns the mission and responsibility of encouraging larger and more effective use of radio spectrum in the public interest to the FCC. To this end, the FCC grants licenses that may be used for purposes of experimentation, product development and market trials, which are not otherwise permitted under existing service rules [8].

These experimental licenses are classified in the five categories $^{5}$ presented in Fig. 1. According to the FCC, this license structure "benefit the development of new technologies, expedite their introduction to the marketplace and unleash the full power of innovators to keep the United States at the forefront of the communications industry." [9]

\footnotetext{
${ }^{3}$ Non-confidential information regarding license assignment can be accessed through the FCC's web-page. Nevertheless, there is no access to a complete database, including technical and non-technical details, for massive consultations of the users under a single repository.

${ }^{4}$ The rules and considerations of the ERS are contained in the Part 5 of the Code of Federal Regulations, Title 47, 2013. [24]

${ }^{5}$ This new license scheme was implemented by the FCC in 2013, where three new types of Experimental Radio Service licenses were included: Program, Medical Testing and Compliance Testing. Since April 2017 the FCC has started to receive applications for Program Licenses in its "FCC's New Experimental Licensing System". [13]
}

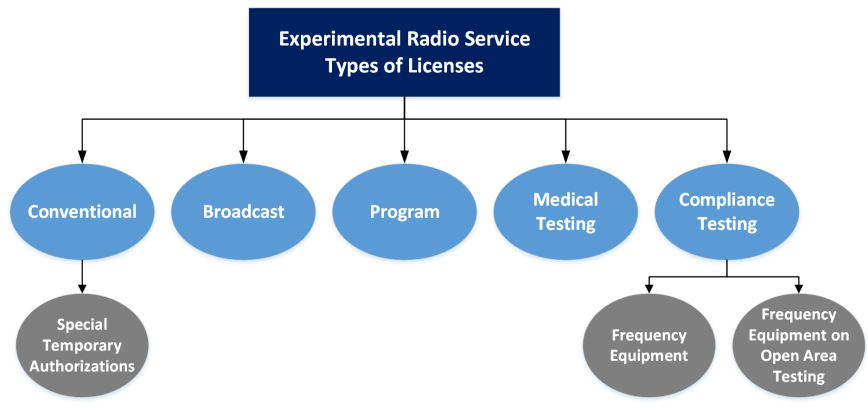

Fig. 1: ERS License Classification

a) Conventional: These licenses are issued for a specific research or experimentation project or a series of closelyrelated projects. However, if the projects are widely divergent and unrelated, experiments must be conducted under separate licenses. In addition, a conventional license could also be granted for a product development trial, or a market trial.

Special Temporary Authorizations (STA) are a subcategory of conventional licenses. An STA applies to cases where applicants need to operate transmitting equipment in circumstances where it is not possible to wait for a license to be issued. This permission is granted when the program is expected to last no more than six months.

b) Broadcast: An authorization is granted for research and experimentation projects whose focus is on the development and advancement of new broadcast technologies, equipment, systems or services. This authorization is limited to utilization by the general public and stations intended for reception only.

c) Program: Intended for qualified institutions wishing to conduct an ongoing program of research and experimentation under a single experimental authorization. These institutions include: colleges, universities, research laboratories, manufacturers and medical research institutions that integrate radio frequency equipment into their end products.

d) Medical Testing: Program authorizations are generally issued for medical research institutions. Nevertheless, a medical testing license is intended for hospitals and health care institutions that demonstrate expertise in testing and operating medical devices using wireless technology in clinical trials for diagnosis, treatment, or patient monitoring.

e) Compliance Testing: This type of license is specific for laboratories recognized by the FCC to perform the following experiments:

- Product testing of radio frequency equipment.

- Testing of radio frequency equipment in an Open Area Test Site ${ }^{6}$.

\footnotetext{
${ }^{6}$ Tests are normally performed in an RF shielded room or anechoic chamber so that the radio signals used to illuminate the product do not radiate over the airwaves. Open area test sites refer to locations where the signals may radiate over the airwaves and pose a significant risk of interference to communications services. [7]
} 


\section{B. Experimental Radio Service Regulation}

The ERS is administered by the FCC's Office of Engineering and Technology (OET) and its Experimental Licensing Branch. The rules in place were adopted after the 2013 Report \& Order (R\&O) (FCC 13-15), where the Commission included numerous changes to its experimental licensing branch, revising and streamlining its rules ${ }^{7}$. With the new rules, the FCC states that the Experimental Radio Service will have "a more flexible framework to keep pace with the speed of modern technological change, while continuing to provide an environment where creativity can thrive" [9].

The rules of the Experimental Radio Service provide great flexibility with regards to the technical parameters and its petitions. Nonetheless, the FCC, as the enforcement agency, clearly mandates that: "radiations of the transmitter shall be suspended immediately upon detection or notification of a deviation from the technical requirements of the station authorization until such deviation is corrected." [8]

In what follows we present the information included in [8] regarding the Experimental Radio Service rules, restrictions and regulation.

a) Allowable Frequency: Frequencies are assigned on a shared basis and not for the exclusive use of any one licensee. All applicants shall ensure that transmitted emissions remain within the authorized frequency band and bandwidth. Under normal operating conditions, licensees could be allowed to utilize any Federal or non-Federal frequency, except for those exclusively allocated to passive services (e.g., radio astronomy and space research services) $)^{8}$.

In the case of Broadcast licenses, the assigned frequencies should be the most suitable for the purpose of the experimentation and those which are the least likely to cause interference to established stations. If an experiment cannot be feasibly conducted on frequencies allocated to a broadcasting service, an experimental station may be authorized to operate on other frequencies showing that the proposed operation can be conducted without causing any harmful interference.

b) Maximum Transmission Power: Since experimental licenses are meant to share spectrum, the transmitting radiated power is understood to be limited to the minimum practical radiated power necessary for the success of the experiment. Furthermore, in the case of broadcast experimental radio stations, the power shall not exceed the maximum power specified by more than five percent. The efficiency factor should be guided by values normally utilized for similar equipment operated within the authorized frequency range.

c) Non-interference Criterion: The FCC mandates that the operation of an experimental radio station is permitted only on the condition that harmful interference is not caused

\footnotetext{
${ }^{7}$ Modifications that have already been included in the Part 5 of the Commission's Rules since January 31, 2013 (Report \& Order (R\&O) FCC 13-15 released by the FCC on February 4th, 2013).

${ }^{8}$ On July 6, 2015, the Commission adopted a Memorandum Opinion and Order modifying its rules covering Experimental Radio Service. The FCC modified its rules to permit certain licenses to use bands exclusively allocated to the passive services in some circumstances for testing medical devices. [1]
}

to any station. The Commission requires that if harmful interference to an established radio service occurs, the experimental licensee should cease transmissions immediately upon becoming aware of such interference. The radio operator is not allowed to resume transmissions until the licensee establishes, to the satisfaction of the Regulator, that further harmful interference will not be caused.

d) Authorized Applicants: Even though the FCC rules provide great flexibility regarding technical parameters, authorizations are issued only to entities qualified to conduct the requested experimental operations. Consequently, it is mandated by the FCC that no experimental radio transmitter, within the United States, could start operations before a proper authorization has been granted. ${ }^{9}$

e) Obtaining a License: One key element in the procedure to obtain an authorization is the usage of FCC's Experimental Licensing Standard Forms, which are submitted electronically through the OET's portal. The Commission seeks to expedite the emitting process and, to assure that only the necessary information is supplied in a consistent manner by the requesters. Each application should be complete with regards to the information required, e.g., transmitter location, equipment, power, operating frequencies, etc.

f) License Period: The license period is another flexible aspect of the Commission's ERS program. For Conventional, Program, Medical and Compliance licenses, the regular term assigned by the FCC is 24 months ( 2 years). However, the applicant can request a license for up to 5 years. Additionally, any license can be renewed for an additional term (no longer than 5 years) upon applicant's request ${ }^{10}$.

\section{Methodology}

The FCC discloses all available information regarding the Experimental Radio Service to the public. Nonetheless, there is no access to a single repository or API for advanced queries ${ }^{11}$. Consequently, the very first step in this work was to scrape the available information to build a single database of technical and non-technical details of the ERS licenses.

\section{A. Scraping the Information}

Experimental Licensing information in the United States is available since 1987. However, the electronic filing of applications started in 1995. Consequently, between 1987 and 1995, most of the application registers do not contain any additional details ${ }^{12}$.

To access the available information any user can utilize the "Generic Search Platform" created by the OET ${ }^{13}$. The

\footnotetext{
${ }^{9}$ One special case is considered under this restriction: a license is not required when the device is fully contained within an anechoic chamber or a Faraday cage.

${ }^{10}$ This does not apply for Special Temporary Authorizations (STA), which maximum duration is 6 always months.

${ }^{11}$ The ERS portal is mainly designed for individual queries about the status of the different applications [19].

${ }^{12}$ This applications only contain licensee name and the FCC identification numbers [18].

${ }^{13}$ Available through the FCC's web page at https://apps.fcc.gov/oetcf/els/ reports/GenericSearch.cfm.
} 


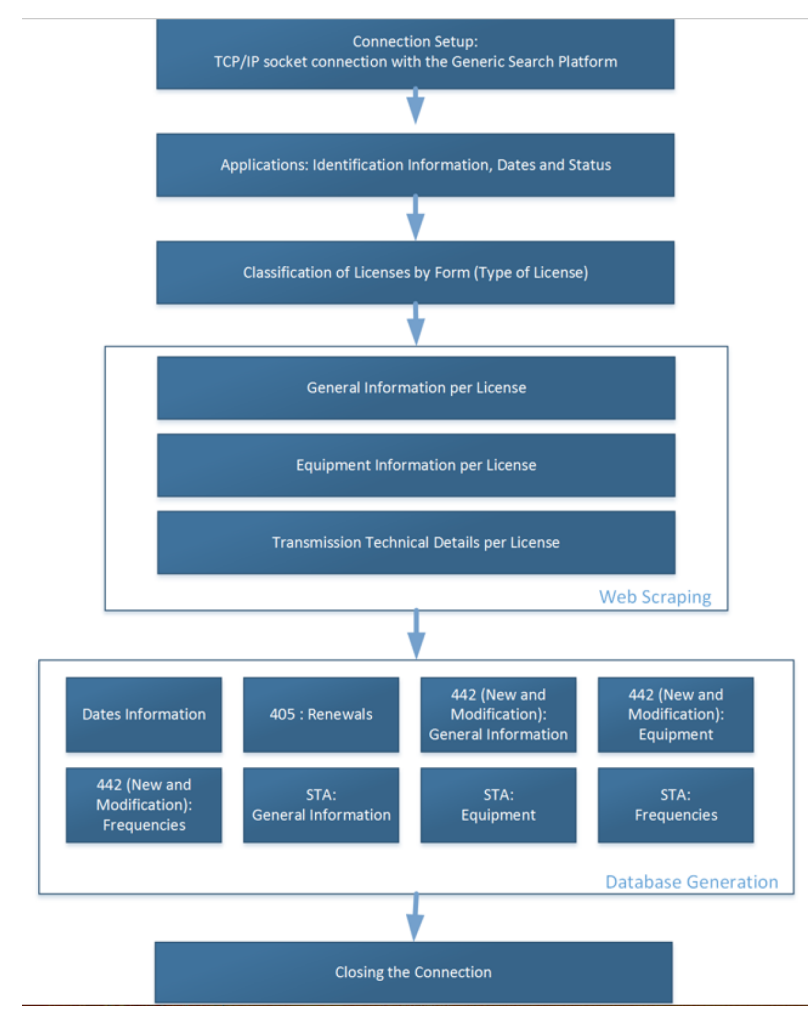

Fig. 2: Scraping Code Process

tool only allows for specific views of the Experimental Licensing Branch information, where the results of each query are presented using HyperText Markup Language (HTML). However, no Application Program Interface (API) is available to directly query the Experimental Licensing Branch database in an automated manner. Consequently, developing a scraping interface was the first step in this work ${ }^{14}$. Its objective is to create automatic processes to search, analyze and transform the unstructured data generated through the OET web search platform as it is shown in Fig. 2.

\section{B. Data Mining}

The main goal of this work is to highlight the data behind ten years of experimental licenses in the United States. We take a step further and try to find potential details and characteristics hidden in those experimental authorizations. This should allow us to achieve a deeper understanding of not only the licenses themselves, but also the applicants and requesting procedures behind them. To fulfill this goal, data mining techniques and models are implemented as follows:

- Predict future values of the experimental licenses based on the scraped and stored historical data. In this manner, the first task will be to produce simple and multi-linear regression models trained using the available information of the past ten years.

\footnotetext{
${ }^{14}$ The scraping interface was implemented as a Java Code for a complete automatic web extracting. Nevertheless, there is a limitation of 200 applications at a time, due to the restrictions of visualization in the Generic Search Platform of the FCC.
}

- Using data mining unsupervised learning techniques such as clustering and classification, we aim to create highlevel views of the applicants and the license requesting procedures. This allows us to, first, asses whether applications are treated differently based on the requested frequency, maximum power or other license parameters. In addition, we seek to identify similar groups of applications based on their technical and non-technical characteristics rather that in the fixed licensee identification mandated by the FCC.

- The FCC requires all applicants of Special Temporary Authorizations to detail the purpose of operation of the requested license [19]. Using this information and text mining techniques we aim at identifying the most common "purpose of operation" of the ERS.

\section{The Numbers Behind the Experimental RAdio SERVICE}

\section{A. Evolution of the Experimental Licenses in the past 30 years}

Since 1987 the FCC has approved 24,950 licenses ${ }^{15}$. As it can be observed in Fig. 3, the number of granted licenses has continuously grown: approved applications have increased from less than 500 until 1998 to more than 2,000 since 2014. However, these licenses are not uniformly distributed over these thirty years. We find that $61 \%$ of licenses have been granted during the past 10 years and, 39\% were emitted just in the period between 2012 and $2016^{16}$.

In this last decade a total of 13,653 experimental authorizations were granted in the United States. The amount of assignations has gone from 743 in 2007 to more than 2,000 licenses by the end of 2016 (See Table I). This increase is especially evident in the number of Special Temporary Authorizations, which presents the most significant growth. Indeed, the number of approved STAs has increased $15 \%$ per year, on average, while other types of licenses have lower growth rates: New (2\%), Modification (10\%) and Renewal $(11 \%)$, as shown in Fig. 4.

\section{B. Processing Delay}

The time elapsed from the date an application is received to the date a license is issued ${ }^{17}$ is considered as the processing time delay of an application. According to the Report of the Unlicensed Devices and Experimental Licenses Working Group of the FCC, "[e]xperimental applications that are solely within the FCC's jurisdiction are usually approved in less than a month". From our gathered historical data we have observed that the average processing time has, in fact, been reduced from over 100 days $^{18}$ in 2007 to an average of 23.31 days in the past year. This decreasing trend in the processing delay of an application is also true for other statistical descriptors. For instance, if we were to observe the median value for

\footnotetext{
${ }^{15}$ Includes: New, Modification, Renewal, Expired, STAs

${ }^{16}$ This license distribution is the reason why, in this work, we focus on the experimental licensing activity occurring in the last ten years.

${ }^{17}$ Within the OET's portal this date is known as "Status Date".

${ }^{18}$ Calendar days: includes weekends and holidays.
} 


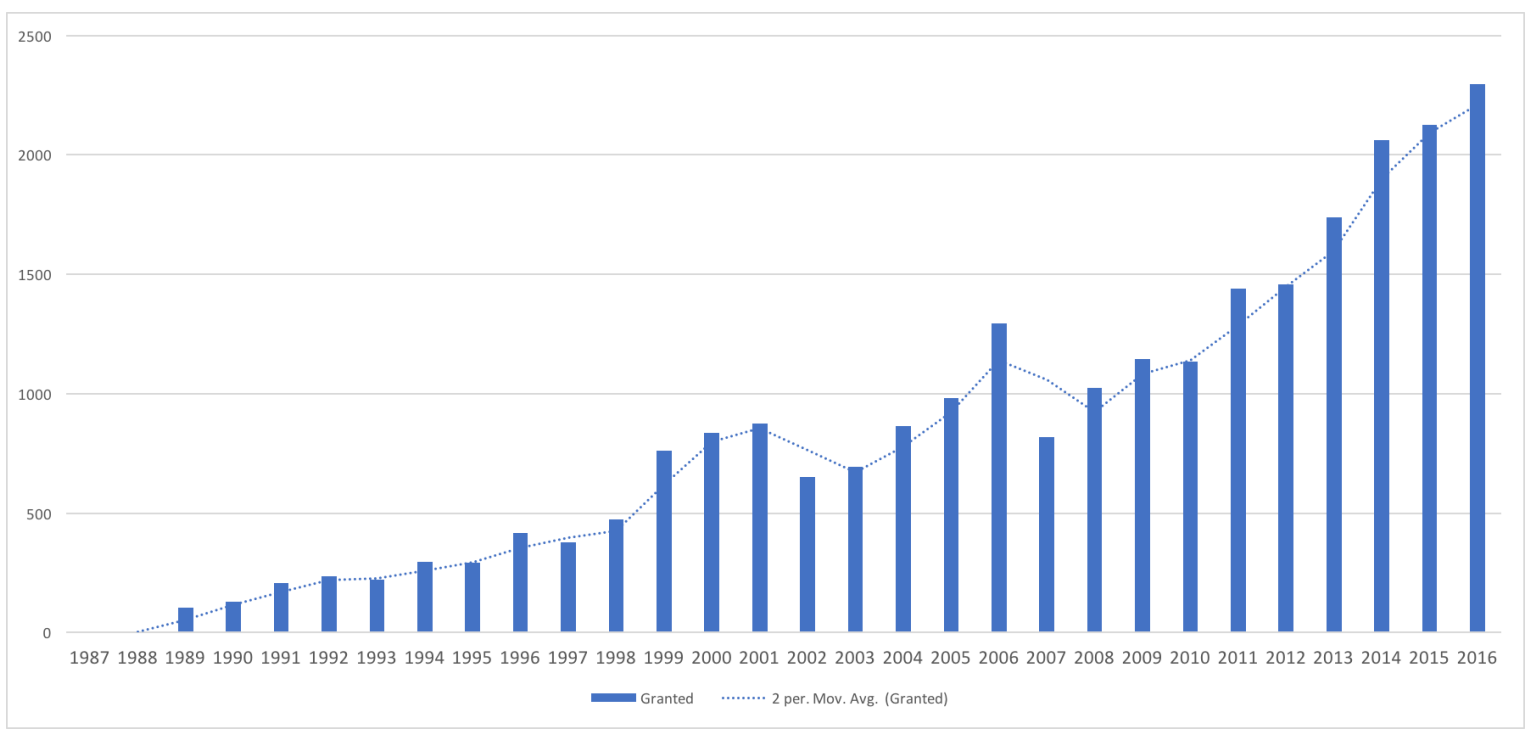

Fig. 3: Experimental Licenses granted in the U.S. since 1987

TABLE I: Evolution in the Number of Experimental Licenses from 2007 to 2016

\begin{tabular}{|l|l|l|l|l|l|l|l|l|l|l|l|}
\hline License & $\mathbf{2 0 0 7}$ & $\mathbf{2 0 0 8}$ & $\mathbf{2 0 0 9}$ & $\mathbf{2 0 1 0}$ & $\mathbf{2 0 1 1}$ & $\mathbf{2 0 1 2}$ & $\mathbf{2 0 1 3}$ & $\mathbf{2 0 1 4}$ & $\mathbf{2 0 1 5}$ & $\mathbf{2 0 1 6}$ & Total \\
\hline New & 221 & 174 & 225 & 207 & 214 & 241 & 319 & 354 & 325 & 327 & 2607 \\
\hline Modification & 38 & 62 & 80 & 74 & 108 & 130 & 126 & 142 & 156 & 271 & 1187 \\
\hline STA & 281 & 337 & 431 & 365 & 544 & 620 & 796 & 779 & 962 & 1130 & 6245 \\
\hline Renewals & 203 & 220 & 413 & 320 & 363 & 354 & 376 & 404 & 506 & 453 & 3614 \\
\hline Total & 743 & 793 & 1149 & 966 & 1229 & 1345 & 1617 & 1679 & 1949 & 2181 & $\mathbf{1 3 6 5 3}$ \\
\hline
\end{tabular}

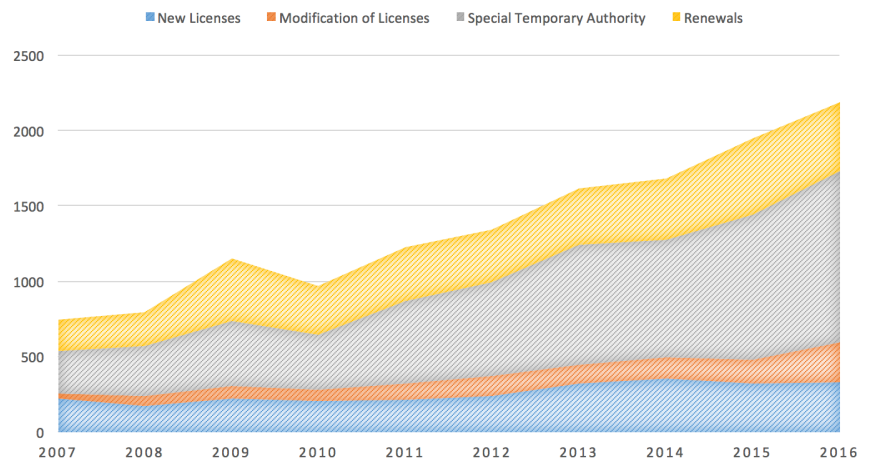

Fig. 4: Evolution in the Number of Experimental Licenses for the past 10 years

the processing time, $50 \%$ of the total applications have been resolved in less than thirty days except for 2007. Furthermore, in most years, the median resolution time is approximately 20 days as it is presented in Fig. 5.

As mentioned in Section V-A most requests for experimental authorizations correspond to Conventional licenses (New and Modification), Special Temporary Authorizations and Renewals. We observe some major variance in the evolution of these licenses, differences that are also shown in their corresponding processing delays. In Fig. 6 we can see that even though the average delay for all of them has been reduced from 2007 to 2016, different delays are experienced by distinct

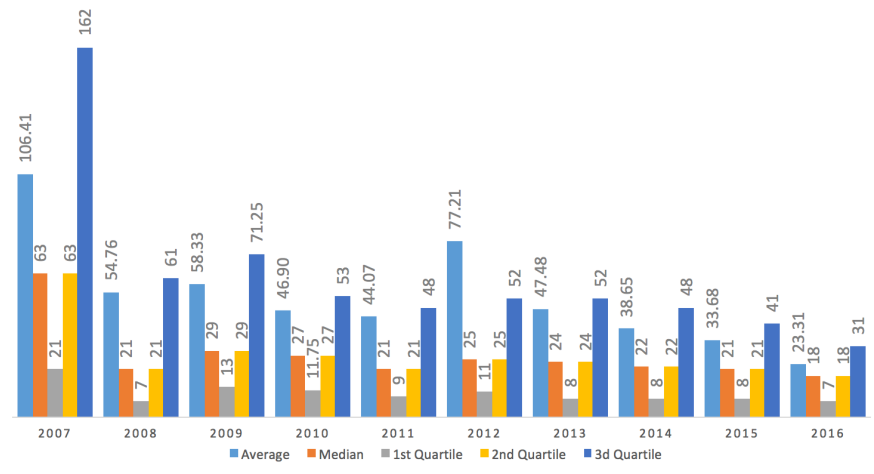

Fig. 5: Processing Delay (Statistical Descriptors)

types of applications. Throughout these 10 years STAs have had the least processing delay, which agrees with the intention of this type of authorizations (See Section III-A). On the other hand, new conventional licenses can take, on average, $68 \%$ more time to be processed than a temporary authorization.

In [10] the FCC's Spectrum Policy Task Force explains that "[t]he principal concern of these parties appeared to be the delays involved in obtaining an experimental authorization due to NTIA coordination...". However, there is not an explicit disclosure of whether coordination with the NTIA was or not necessary for the application process within the available information. Consequently, to analyze the implications in the processing time when the NTIA is involved, we filtered all 


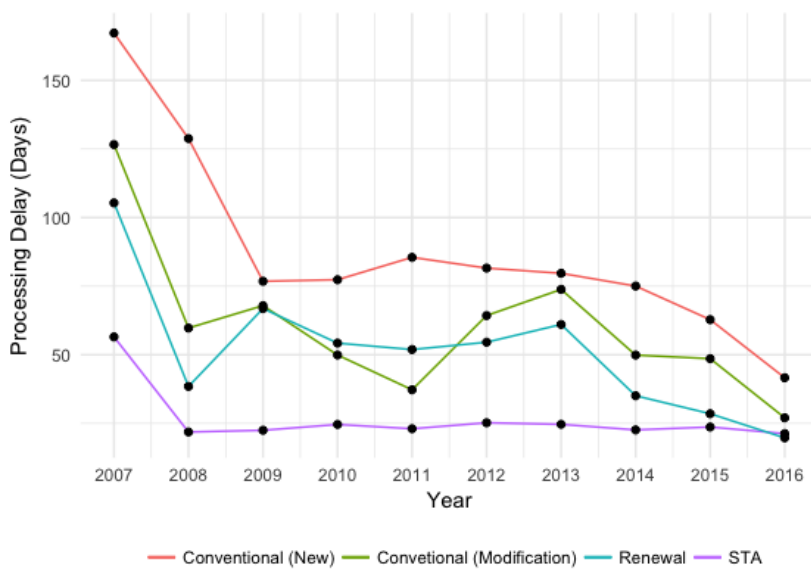

Fig. 6: Processing Delay by Type of Application

federal exclusive allocations between $300 \mathrm{MHz}$ and $3 \mathrm{GHz}^{19}$. This allowed us to compare between the average and median processing times depicted in Fig. 7. As it is shown when the NTIA is involved, the average and median delays are always higher, almost double, than the rest of applications for experimental licenses. However, it is necessary to point that these delays have also been considerably reduced to an average of 42 and a median of 35 days during last year.
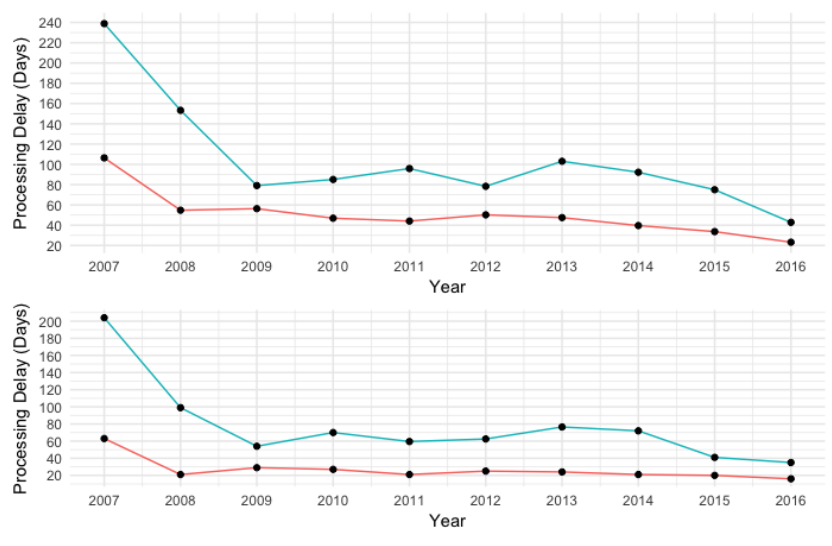

— General - NTIA Applications

Fig. 7: Processing Time Delay when the NTIA is involved (Top: Average, Bottom: Median)

\section{Conventional Licenses}

So far we have pointed out the main findings with regards to all applications presented for experimental licenses. Nevertheless, in the following sections we will turn our attention to the analysis of the most common types of licenses: conventional and temporary authorizations. ${ }^{20}$

\footnotetext{
${ }^{19}$ Corresponds to the UHF band, which is the frequency range with the greatest number of assignations (See Section V-C3b)

${ }^{20}$ Licenses for Program, Medical Device Testing, and Compliance Testing were introduced in 2013 [9]. Moreover, Program licenses were recently included (April 2017) as part of the application process of the FCC [13].
}

1) Types of Licensees: The Commission requires all license requesters to identify themselves as part of the application process. Thus, a licensee could be classified into one of these five categories: Individual, Association, Partnership, Corporation and Other. For the past 10 years most of the licenses have been issued for applicants identified as corporations, corresponding to $86 \%$ of the total licenses (Fig. 8). On the other hand, the ratio of applicants identified as individuals and partnerships correspond to just $3 \%$ and $2 \%$, respectively. Licensees classified as "Others" accounts for $9 \%$ of the licensees. This classification was introduced by the FCC to allow the applicant to describe "what best fits its identity" [19]. The most commonly utilized descriptors are:

- Governmental Institutions:

- City Agencies

- State Agencies

- Port Authority

- Colleges and Universities

- Research Organizations and Laboratories

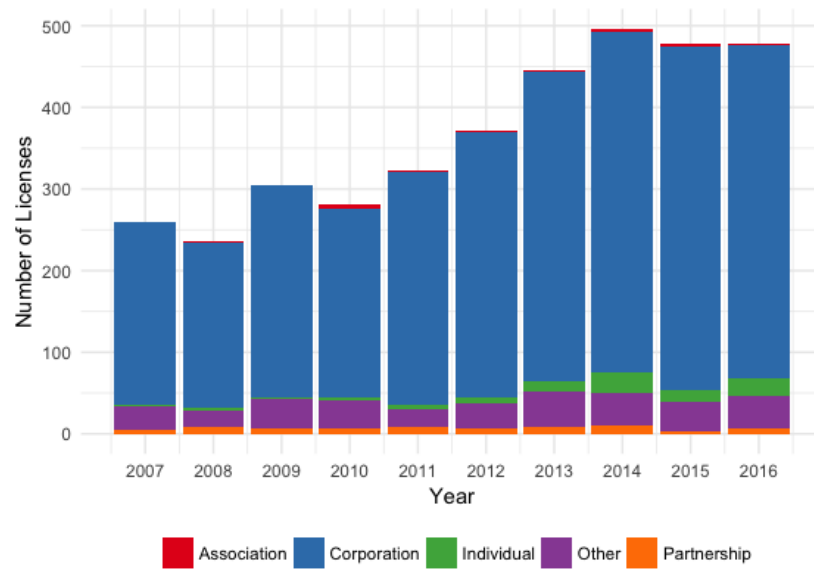

Fig. 8: Conventional: Number of Licenses by Applicant Type

2) License Duration: A conventional experimental license could be issued for any period between 1 month and 5 years. In the last decade, $69 \%$ of the conventional licenses are authorized for a period of 24 months (See Fig. 9). All applications (except "Association") follow a similar distribution with regards to the duration of the license, where the peak is always located around 2 years and there is a high concentration of authorizations around 12 months.

3) Technical Parameters: It is well known that at the core of any FCC license lie its authorized technical parameters. This is not the exception for Experimental Radio Service authorizations. In this light, we will conclude the analysis of ERS conventional licenses by exploring its utilized equipment, allocated frequencies and maximum allowable power ${ }^{21}$.

a) Equipment: One of the main components of any ERS application is the list of all radio equipment to be

\footnotetext{
${ }^{21}$ As exposed in Section III-B, one of the main claims made by the FCC is the "great flexibility" towards authorized technical parameters.
} 


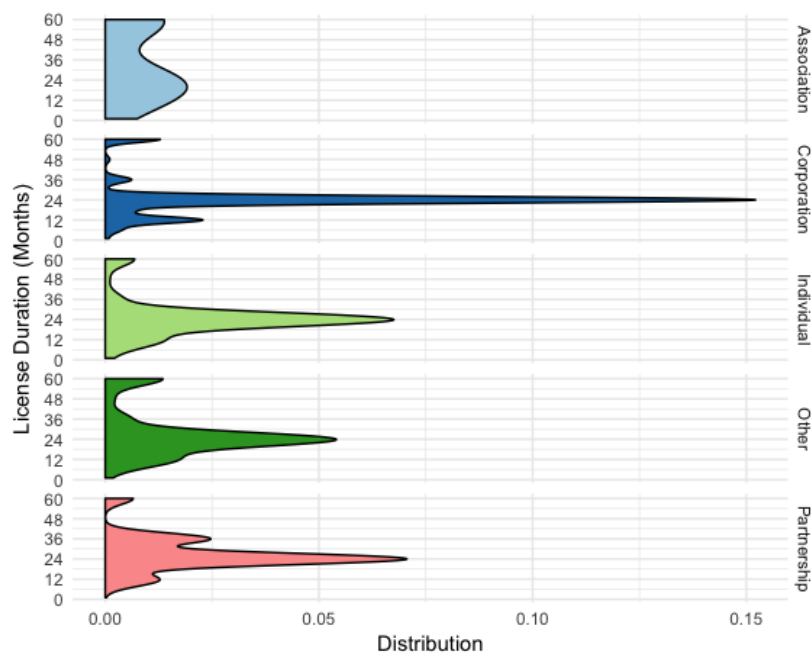

Fig. 9: Conventional: License Duration Distribution

used in the experiment. This list includes the manufacturer of the transmitter, model number and the total amount of units to be utilized. As part of the requirements, the FCC also mandates the inclusion of an experimental identification, which differentiates between commercial and research units. Using the latter, we were able to determine the amount of "de facto experimental equipment" being deployed. Our analysis showed that, on average, only $40 \%$ of the total equipment is experimental. This low ratio is more evident in the case of corporations, the most common licensee, with only $35 \%$ of experimental equipment. On the other hand, partnerships and individuals have utilized at least $50 \%$ of experimental units in their deployments, the highest among all other type of licensee (See Fig. 10).

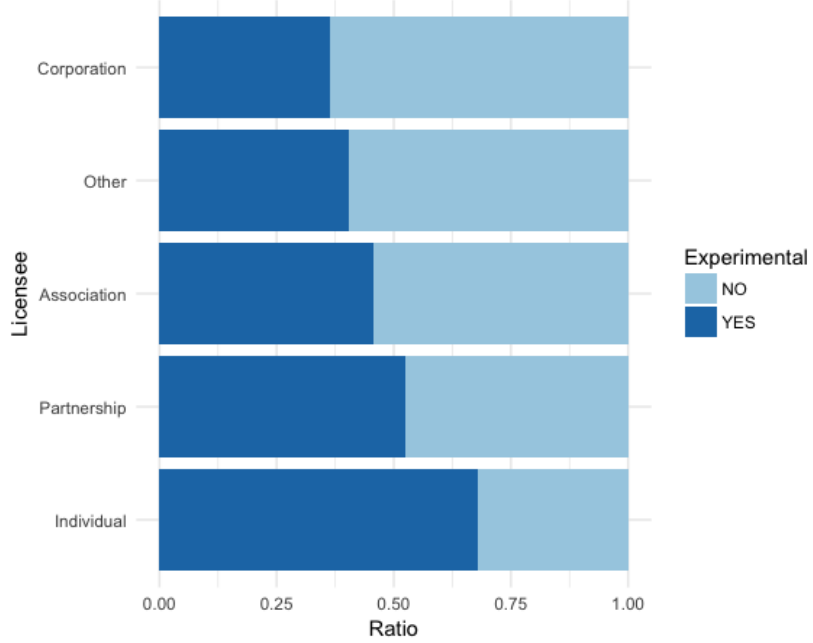

Fig. 10: Conventional: Experimental Equipment Distribution

b) Frequency: Rules within the ERS mandate that licenses could be allowed to utilize any Federal and non-Federal frequency, except for those exclusively allocated to passive services $^{22}$. This great flexibility is evident in these past 10 years. Frequencies have been assigned in all radio bands defined by the International Telecommunications Union (ITU) [11] with a total of 76,611 frequencies authorized since 2007 (See Table II). Within this wide range of used frequencies we can see that the most common bands for conventional licenses are in the Ultra High Frequency (UHF) and Super High Frequency (SHF) range, with 37,069 and 15,806 frequencies, respectively (See Fig. 12). These results are consistent with the general distribution of frequencies depicted in Fig. 11. Note that, while the distribution itself has changed, most of the authorizations are always concentrated between $10 \mathrm{MHz}$ and $10 \mathrm{GHz}$, whit the greatest density of utilized frequencies located within the UHF and SHF bands.

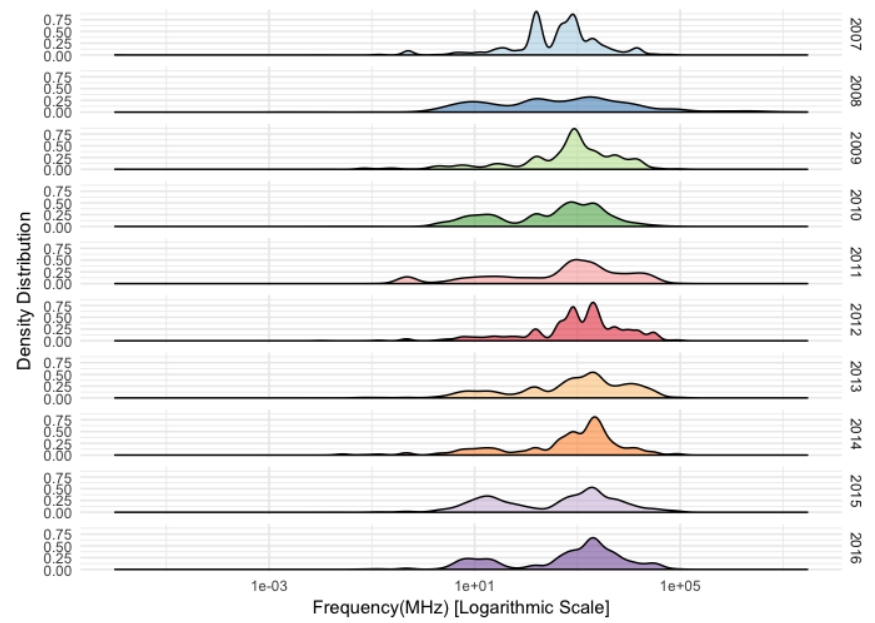

Fig. 11: Conventional: Frequency Distribution

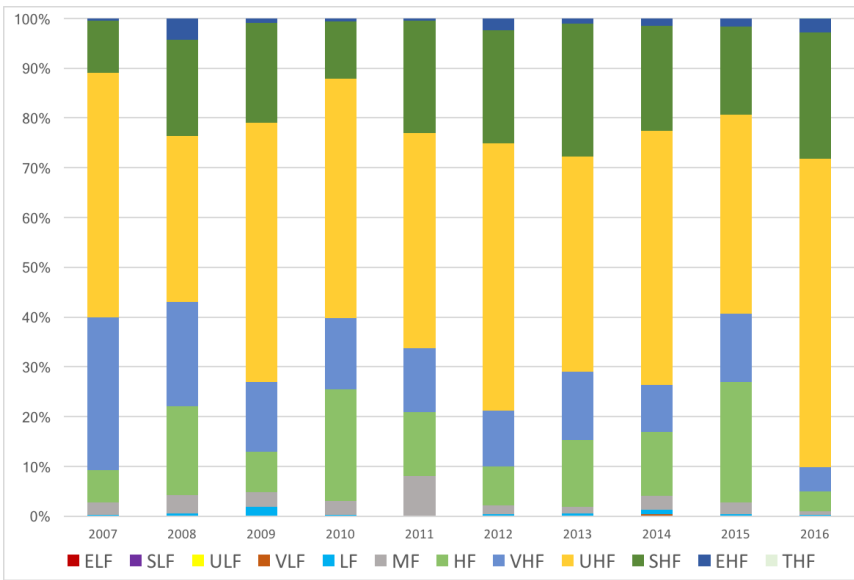

Fig. 12: Conventional: Frequency Distribution by Radio Band

Either due to the imminent spectrum scarcity conditions or the advance of telecommunications, the wireless community has shown increased concern about the unused resource availability in very high frequency bands [6], [21], [25].

\footnotetext{
${ }^{22}$ Certain licenses can utilize the bands exclusively allocated for passive services in some circumstances for testing medical devices [1].
} 
TABLE II: Conventional: Frequency Distribution By Radio Band

\begin{tabular}{|c|c|c|c|c|c|c|c|c|c|c|c|}
\hline Band & 2007 & 2008 & 2009 & 2010 & 2011 & 2012 & 2013 & 2014 & 2015 & 2016 & TOTAL \\
\hline ELF & 0 & 2 & 0 & 0 & 0 & 0 & 0 & 0 & 0 & 0 & 2 \\
\hline SLF & 0 & 1 & 0 & 0 & 0 & 0 & 0 & 0 & 0 & 0 & 1 \\
\hline ULF & 0 & 0 & 0 & 0 & 0 & 1 & 0 & 1 & 0 & 0 & 2 \\
\hline VLF & 1 & 0 & 2 & 5 & 0 & 9 & 1 & 41 & 6 & 1 & 66 \\
\hline LF & 11 & 14 & 79 & 8 & 7 & 19 & 56 & 99 & 37 & 29 & 359 \\
\hline MF & 113 & 118 & 137 & 177 & 687 & 112 & 111 & 306 & 269 & 75 & 2105 \\
\hline HF & 294 & 581 & 359 & 1387 & 1098 & 497 & 1249 & 1433 & 2752 & 462 & 10112 \\
\hline VHF & 1395 & 678 & 627 & 893 & 1091 & 730 & 1276 & 1057 & 1563 & 554 & 9864 \\
\hline UHF & 2229 & 1084 & 2330 & 2984 & 3698 & 3457 & 4027 & 5678 & 4559 & 7023 & 37069 \\
\hline SHF & 472 & 625 & 898 & 713 & 1918 & 1459 & 2482 & 2348 & 2016 & 2875 & 15806 \\
\hline EHF & 23 & 140 & 42 & 39 & 46 & 153 & 97 & 175 & 194 & 308 & 1217 \\
\hline THF & 0 & 0 & 0 & 0 & 0 & 0 & 0 & 0 & 0 & 8 & 8 \\
\hline TOTAL & 4538 & 3243 & 4474 & 6206 & 8545 & 6437 & 9299 & 11138 & 11396 & 11335 & 76611 \\
\hline
\end{tabular}

This fact is also reflected in the evolution of conventional experimental licenses in the last decade. Thus, we can see that the exploration of bands over $5 \mathrm{GHz}$ has definitely had a surge since 2011, as it is depicted in Fig. 13. Within this shift in requested frequencies, the exploration of bands around 35 and $45 \mathrm{GHz}$ is, without a doubt, the frequency range showing the most notable increase.

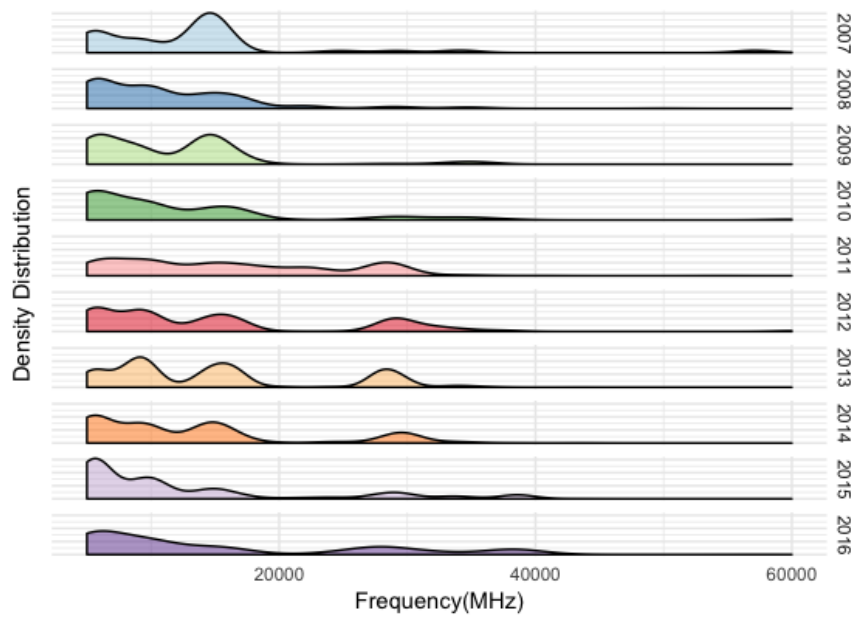

Fig. 13: Conventional: Frequency Distribution Above $5 \mathrm{GHz}$

c) Authorized Power: To conclude this technical analysis we will explore the maximum authorized power levels in conventional licenses. In the same way as allowable frequency, power authorizations are also very flexible. The maximum authorized transmission power varies from very low values, i.e., less than $1 \mathrm{uW}$, to significantly high power authorizations in the order of GigaWatts. Nevertheless, since 2013 there has been a bigger concentration of authorized powers between $100 \mathrm{~mW}$ and $1 \mathrm{KW}$, contrary to a more uniform distribution in previous years. In fact, $65 \%$ of the experimental stations have operated in this power range (Fig. 14) for the past four years.

\section{Special Temporary Authorizations}

The FCC allows license requesters to obtain an especial category of conventional licenses called Special Temporary

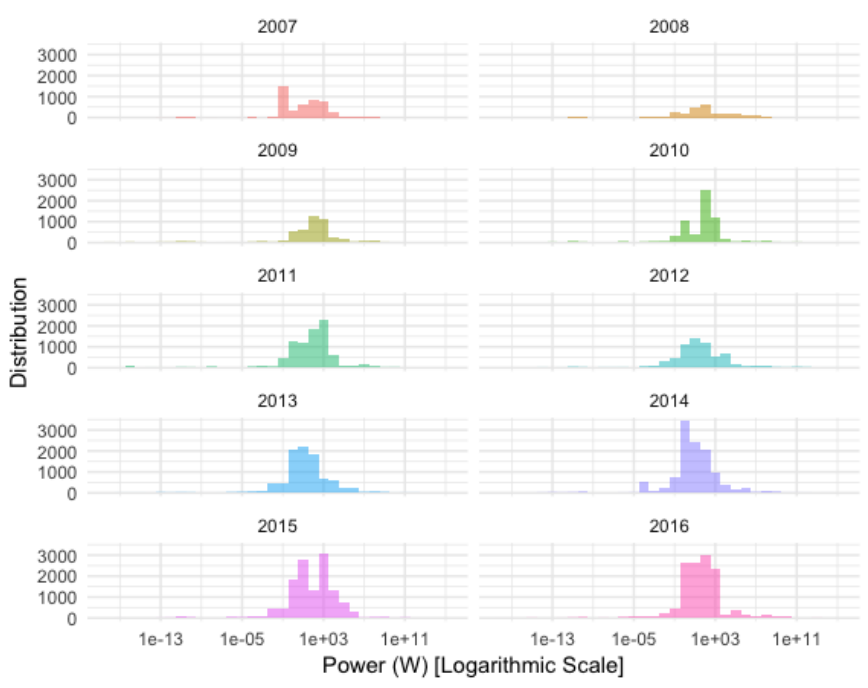

Fig. 14: Conventional: Power Distribution

Authorizations. This type of license applies in cases where requesters need to operate transmitting equipment under especial circumstances and with an experiment duration of less than 6 months [19]. STAs are of particular interest because they have had the biggest growth in the past ten years with an average increase rate of $15 \%$ per year (See Table I). Thus, temporary licenses have increased from only 281 to more than a thousand by the end of 2016, totaling 6,245 authorizations since 2007.

1) License Duration: A Special Temporary Authorization can last anywhere between one day and six months. In the case of the last decade, however, almost half $(47 \%)$ of licenses were approved to last one month or less (See Fig. 15). This trend is yet more salient in recent years (2013-2016) with an average of 56\% licenses granted for that period. Going deeper in the analysis of these short term licenses, we can observe that are authorized for less than three days, $10 \%$, or less than a week (7 days), 52\%. This is portrayed in Fig. 16. This short term authorizations could be directly tied to the purpose of operation of most STAs, which will be explored in section V-D3. 


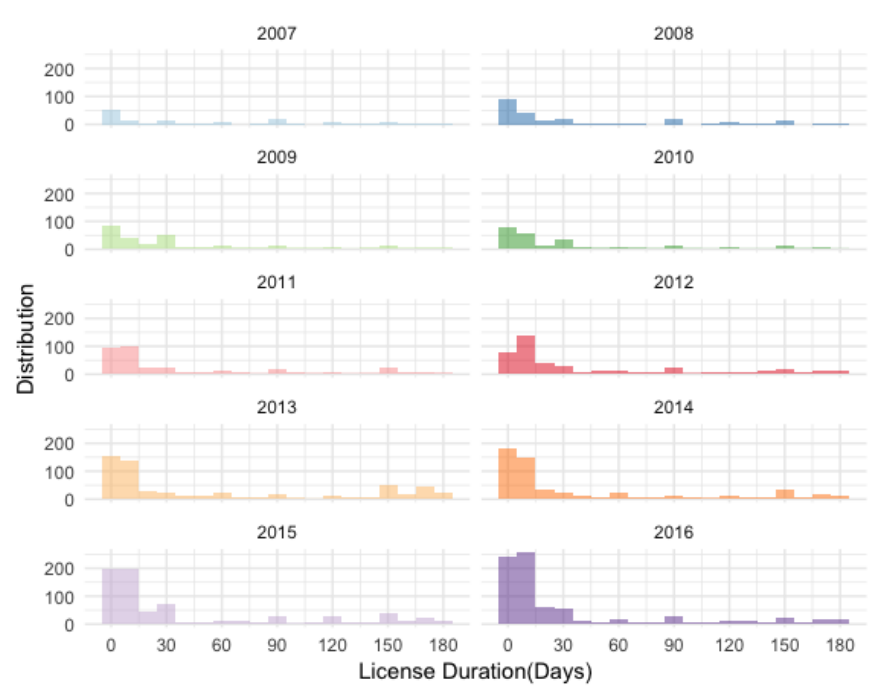

Fig. 15: STA: License Duration Distribution

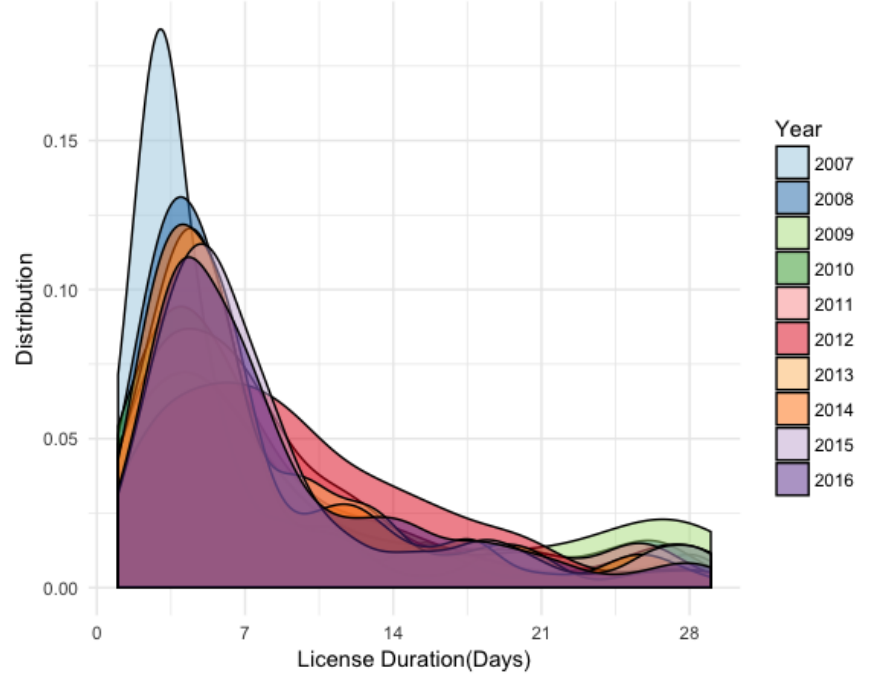

Fig. 16: STA: License Duration Distribution (30 days or less)

2) Technical Parameters: Along the same lines as our conventional licenses analysis, we now analyze the implemented equipment, utilized frequencies and maximum deployed power of the Special Temporary Authorizations that have been granted since 2007.

a) Equipment: Temporary Authorizations are also required to list the complete radio equipment to be used. We find that the average "non-commercial" instrumentation being utilized is only 23\% (Fig. 17). This ratio is significantly lower compared to conventional licenses (40\% Experimental Equipment). One of the main reasons for this phenomenon is also closely tied to the purpose of operation of the majority of temporary authorizations (See Section V-D3).

b) Frequency: Temporary Authorizations are characterized for having great flexibility in its frequency allocation. They span across the majority of ITU Radio Bands [11] with

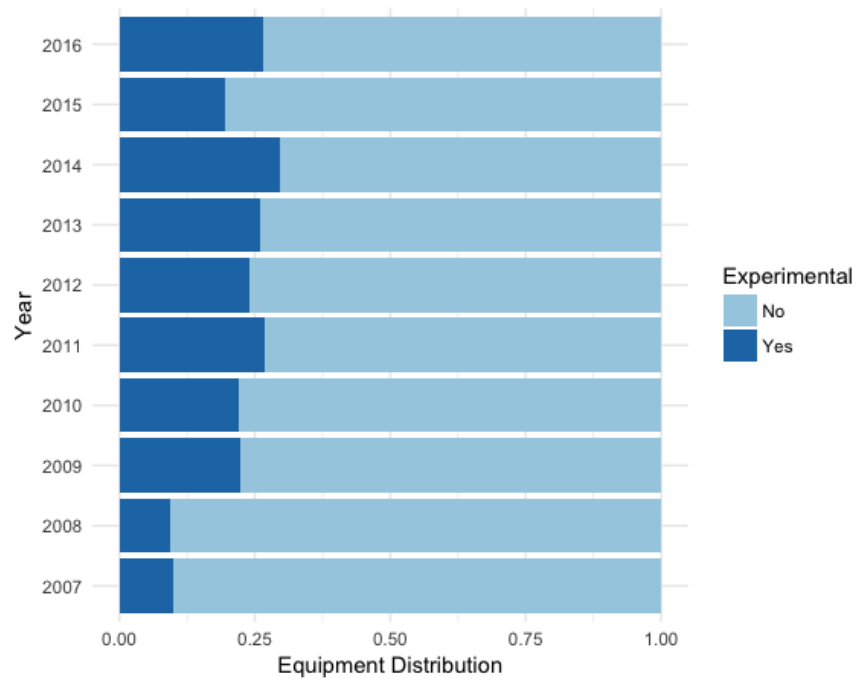

Fig. 17: STA: Experimental Equipment Distribution

a total of 54,764 frequencies. Indeed, the only radio band where zero authorizations have occurred is the ULF (Ultra Low Frequency) band, which ranges from 0.3 to $3 \mathrm{KHz}$ ( See Table III). In the same way as conventional licenses, most of the assigned frequencies correspond to the range between 300$3000 \mathrm{MHz}$ (UHF) with a total of 36,999 (83.34\%) frequencies as shown in Fig. 18.

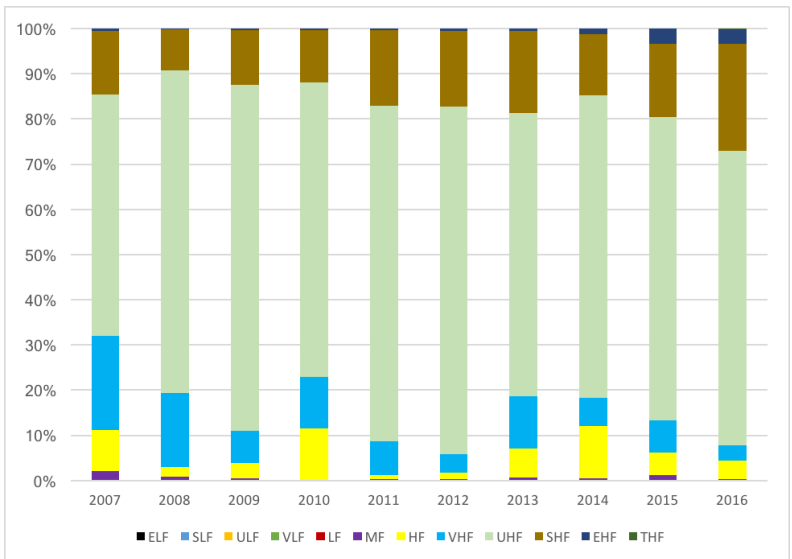

Fig. 18: STA: Frequency Distribution by Radio Band

Regarding the distribution of these frequencies in absence of a fixed band classification ${ }^{23}$, the first thing to mention is that in every year the distribution has been almost identical (Fig. 19). Most of the authorizations are always concentrated between $10 \mathrm{MHz}$ and $10 \mathrm{GHz}$ with their peaks located within the UHF and VHF bands. Additionally, it is interesting to note the movement of the distribution towards higher bands for the past 6 years. We observe a significant amount of authorizations in frequencies such as $38 \mathrm{GHz}, 48 \mathrm{GHz}$ and above.

c) Authorized Power: To conclude the technical parameters section, we will discuss power authorizations in STAs. The

\footnotetext{
${ }^{23}$ Frequency was previously analyzed using the ITU's band classification.
} 
TABLE III: STA: Frequency Distribution by Radio Band

\begin{tabular}{|c|r|r|r|r|r|r|r|r|r|r|c|}
\hline Frequency & $\mathbf{2 0 0 7}$ & $\mathbf{2 0 0 8}$ & $\mathbf{2 0 0 9}$ & $\mathbf{2 0 1 0}$ & $\mathbf{2 0 1 1}$ & $\mathbf{2 0 1 2}$ & $\mathbf{2 0 1 3}$ & $\mathbf{2 0 1 4}$ & $\mathbf{2 0 1 5}$ & $\mathbf{2 0 1 6}$ & TOTAL \\
\hline ELF & 0 & 2 & 0 & 0 & 0 & 0 & 0 & 0 & 0 & 0 & $\mathbf{2}$ \\
\hline SLF & 1 & 0 & 0 & 0 & 0 & 0 & 0 & 0 & 0 & 0 & $\mathbf{1}$ \\
\hline ULF & 0 & 0 & 0 & 0 & 0 & 0 & 0 & 0 & 0 & 0 & $\mathbf{0}$ \\
\hline VLF & 0 & 0 & 0 & 0 & 0 & 0 & 1 & 0 & 1 & 1 & $\mathbf{3}$ \\
\hline LF & 0 & 0 & 0 & 1 & 2 & 0 & 0 & 11 & 13 & 8 & $\mathbf{3 5}$ \\
\hline MF & 60 & 21 & 15 & 6 & 12 & 17 & 49 & 20 & 84 & 18 & $\mathbf{3 0 2}$ \\
\hline HF & 268 & 62 & 121 & 435 & 34 & 69 & 450 & 774 & 436 & 343 & $\mathbf{2 9 9 2}$ \\
\hline VHF & 621 & 461 & 245 & 438 & 308 & 197 & 809 & 427 & 615 & 383 & $\mathbf{4 5 0 4}$ \\
\hline UHF & 1583 & 2021 & 2649 & 2510 & 3007 & 3668 & 4399 & 4492 & 5841 & 6829 & $\mathbf{3 6 9 9 9}$ \\
\hline SHF & 412 & 252 & 414 & 440 & 682 & 796 & 1277 & 909 & 1409 & 2517 & $\mathbf{9 1 0 8}$ \\
\hline EHF & 18 & 7 & 14 & 16 & 14 & 27 & 31 & 82 & 287 & 313 & $\mathbf{8 0 9}$ \\
\hline THF & 0 & 0 & 0 & 0 & 0 & 0 & 1 & 0 & 0 & 8 & $\mathbf{9}$ \\
\hline TOTAL & $\mathbf{2 9 6 3}$ & $\mathbf{2 8 2 6}$ & $\mathbf{3 4 5 8}$ & $\mathbf{3 8 4 6}$ & $\mathbf{4 0 5 9}$ & $\mathbf{4 7 7 4}$ & $\mathbf{7 0 1 7}$ & $\mathbf{6 7 1 5}$ & $\mathbf{8 6 8 6}$ & $\mathbf{1 0 4 2 0}$ & $\mathbf{5 4 7 6 4}$ \\
\hline
\end{tabular}

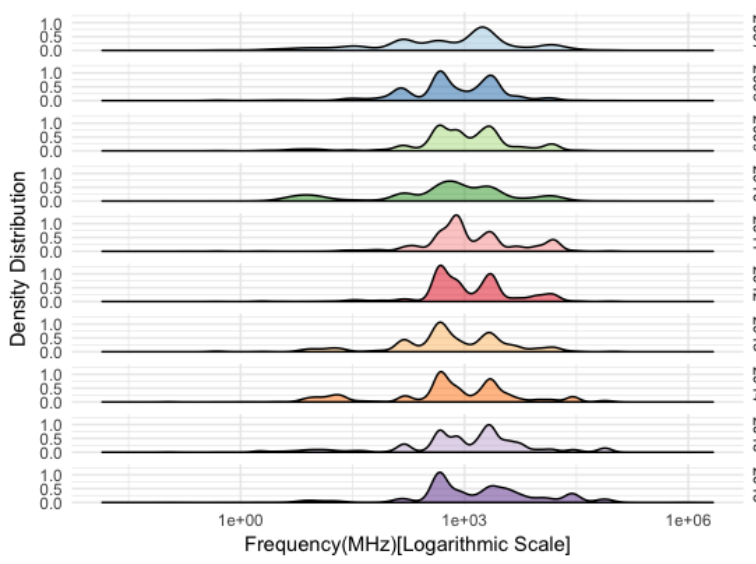

Fig. 19: STA: Frequency Distribution

maximum allowable power levels have varied from very low (less than $1 \mathrm{~mW}$ ) to very high levels (more than $1 \mathrm{GW}$ ) throughout the past 10 years. Furthermore, within this distribution it is interesting to see that the highest concentration of power levels is located between $100 \mathrm{~mW}$ to $1 \mathrm{KW}$, which corresponds to $78 \%$ of the assignments. Nonetheless, we notice a change in the distribution in 2013. Indeed, a higher concentration of frequencies with authorized powers of $100 \mathrm{~mW}$ or less is now more common. Further, this trend not only has been present ever since, but also it has been more evident in the past three years (See Fig. 20).

3) Purpose of Operation: A key characteristic of the STAs is that the purpose of operation of the license should be detailed as part of the application. It is defined as "[a] detailed explanation of the type of operation that will be performed with the ERS license" [19]. In the case of STAs that have been approved since 2007, we can observe a great variety of operational goals. Applications range from very specific deployments and demonstrations for military equipment ${ }^{24}$ to large and complex tests of aircraft systems ${ }^{25}$. Nevertheless, most licenses are authorized for the coverage and support of televised events nationwide, with $73 \%$ of all STAs approved in

\footnotetext{
${ }^{24}$ Federal and Private Companies participate in this type of demonstrations.

${ }^{25}$ One of the companies with the greatest number of licenses is The Boeing Company.
}

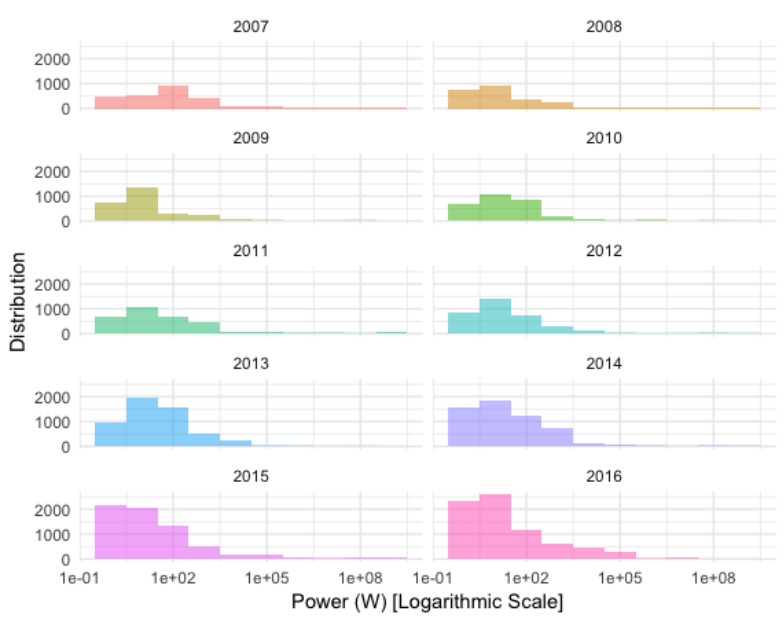

Fig. 20: STA: Power Distribution

the past ten years. These spectrum authorizations are utilized for several activities such as equipment testing, support and coordination of transmissions, personal communications, etc. (Fig. 21). Most of the covered events are sports-related; however, other types of events such as presidential debates ${ }^{26}$ or award ceremonies ${ }^{27}$ also rely on STAs for transmission and support .

On the other hand, temporary authorizations that are not related to televised events, $27 \%$ of all licenses, also cover a wide range of goals. As shown in Fig. 21, they are mainly used for military, government and research projects. Based on the activities detailed in their applications, we can further classify them in: Demonstrations, Developing, Analysis, and Testing of wireless technologies.

In this stage of our work we have presented some interesting findings about the Experimental Radio Service of the FCC. First, from an applicant perspective, we find that experimental licenses are indeed characterized by a great flexibility in terms of technical parameters. This is reflected on authorizations of a wide range of equipment, frequency, and power. From the regulatory point of view, we noted that even though the

\footnotetext{
${ }^{26}$ Classified as Political in Fig. 21.

${ }^{27}$ Classified as Cultural in Fig. 21
} 


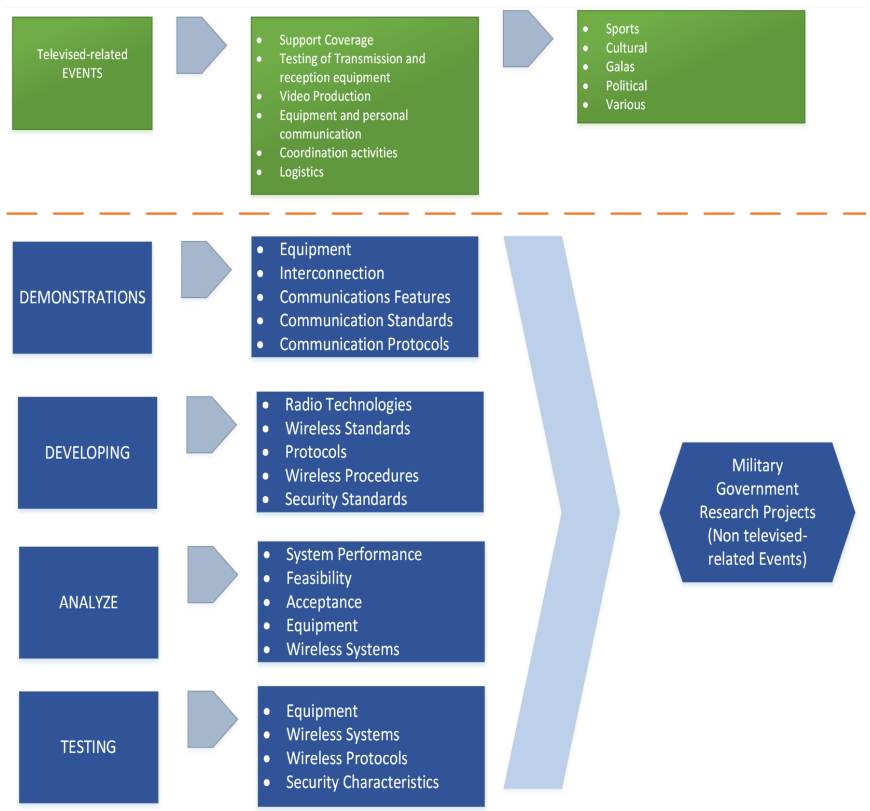

Fig. 21: STA: Purpose of Operation

number of requesters has increased, especially since 2014, the processing delay has considerably been reduced. In terms of the operators of these licenses, corporations are the entities requesting the largest number of experimental licenses. In addition, an important result in the case of Special Temporary Authorizations is the fact that most licenses are granted for the coverage and support of televised events.

\section{DeEPer Data Exploration}

In the previous sections we explained the basic features concerning the Experimental Radio Service of the FCC. However, for a deeper understanding of these licenses, we find it important to explore potential details that might be hidden within a decade of experimental authorizations.

\section{A. Predicting Processing Times}

One of the main concerns when obtaining an experimental license is the time burden attached to this process. As presented in Section V-B this time has already been reduced, going from an average 100 days in 2007 to less than a month in 2016. Nevertheless, using the scrapped data we not only can describer these past details, but also try to predict future delays in getting an experimental authorization.

a) Simple Linear Regression: We will start this mining section with the a very straightforward model: a simple linear regression between year and processing time (See Fig. 22). The constructed model is capable of accounting for $84.11 \%$ of the variance from one year to the following $\left(R^{2}\right)$. This high accountability is also shown in the adjusted $-R-$ square $^{28}$ with an $81,85 \%$ of the delay variance. Moreover, we can

\footnotetext{
${ }^{28}$ The Adjusted $-R^{2}$ weights the number of variables in the prediction for a more realistic model.
}

observe that the model is statistically significant with low pvalue and t-values (See Table IV).

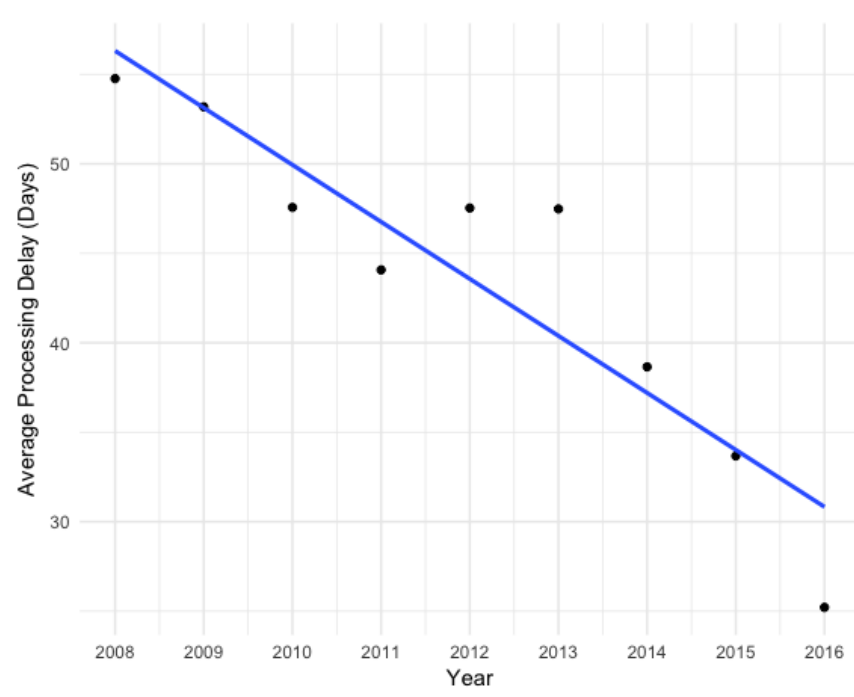

Fig. 22: Linear Relationship Year vs. Processing Delay

TABLE IV: Simple Regression Model Metrics

\begin{tabular}{|c|c|}
\hline Coefficient & -3.18 \\
\hline Intercept & 81.78 \\
\hline R-squared & 0.8411 \\
\hline $\begin{array}{c}\text { Adjusted } \\
\text { R-Squared }\end{array}$ & 0.8184 \\
\hline p-value & 0.0004974 \\
\hline t-value & 0.0000474 \\
\hline
\end{tabular}

The results of the model determine that AverageProcessingDelay $=81.78-3.18 \times$ Year, which implies a reduction of 3.18 days in the processing delay of an application for every new year ${ }^{29}$. Nonetheless, it is necessary to point out that this trend will eventually stabilize $^{30}$, since this model represents a real life scenario that has its own technological and human considerations behind it.

For a better evaluation of the model we compare it to scrapped information of experimental licenses from the year $2017^{31}$, where the average processing delay, so far, has been 19.98 days. If we were to calculate the predicted processing time using our model, it results in a delay of 20.13 days. This shows an error of $0.75 \%$ between the predicted and actual values for the year 2017. Further, in 2018, if the curve has not stabilized yet, we can expect a processing delay of just 16.95 days.

b) Multiple Linear Regression: We first developed a simple model considering only one variable, Year. However, for a more complete analysis we note that it is necessary to

\footnotetext{
${ }^{29}$ The residual Average Standard Error is equal to 4.054 Days.

${ }^{30} \mathrm{No}$ additional reduction in the average processing time.

${ }^{31}$ Information from 2017 corresponds to the period between January 1st to May 31st obtained using the scrapping tool described in Section IV-A Further, these data points were not included in the construction of the simple linear regression model.
} 
include additional factors as independent variables in a more complex multiple linear model. These additional variables include licensee details such as requester classification, license parameters, i.e., duration and type of equipment, and technical parameters, in particular, frequency ${ }^{32}$ and maximum power (See Table V for more details).

TABLE V: Multiple Linear Regression Independent Variables

\begin{tabular}{|l|l|}
\hline \multicolumn{1}{|c|}{ Factor } & \multicolumn{1}{c|}{ Levels } \\
\hline Calendar Year & $2007-2016$ \\
\hline Licensee Type & $\begin{array}{l}\text { Corporation, Individual, Partnership, } \\
\text { Association and Other }\end{array}$ \\
\hline License Duration & $1-60$ months \\
\hline Experimental Equipment & Yes or No \\
\hline Type of Station & FX, MO or FX and MO \\
\hline Number of Frequencies & $1-1000$ \\
\hline Average Power & $1-100000 \mathrm{~W}$ \\
\hline
\end{tabular}

As exposed in the summary of this new model (Fig. 23), the results are not statistically significant. The model cannot be utilized for predicting the delay with any confidence. Indeed, it only accounts for $10 \%$ of the total predictions with statistical insignificance of almost all independent variables ${ }^{33}$.

$\begin{array}{lrrrr}\begin{array}{l}\text { Residuals: } \\ \text { Min }\end{array} & 1 Q & \text { Median } & 3 Q & \text { Max } \\ -128.55 & -42.79 & -18.34 & 17.54 & 1624.09\end{array}$

\begin{tabular}{|c|c|c|c|c|c|}
\hline & Estimate & Std. Error & t value & $\operatorname{Pr}(>|t|)$ & \\
\hline (Intercept) & 68.250862 & 86.061445 & 0.793 & 0.4278 & \\
\hline Year & -7.458786 & 0.495859 & -15.042 & $?<2 \mathrm{e}-16$ & $5 * *$ \\
\hline Application_TypeCorporation & 26.229973 & 19.489718 & 1.346 & 0.1784 & \\
\hline Application_TypeIndividual & 16.232558 & 21.180679 & 0.766 & 0.4435 & \\
\hline Application_Type0ther & 25.842904 & 19.967245 & 1.294 & 0.1957 & \\
\hline Application_TypePartnership & 19.939121 & 21.939422 & 0.909 & 0.3635 & \\
\hline Duration & 0.711167 & 0.127936 & 5.559 & $2.94 \mathrm{e}-08$ & $* * *$ \\
\hline ExperimentalNO & 33.806239 & 79.991633 & 0.423 & 0.6726 & \\
\hline ExperimentalYES & 23.214158 & 79.995056 & 0.290 & 0.7717 & \\
\hline License_TypeNEW LICENSE & 23.629926 & 3.160970 & 7.476 & $9.84 \mathrm{e}-14$ & $+* *$ \\
\hline TypeFX & 6.810745 & 23.169980 & 0.294 & 0.7688 & \\
\hline Tурем0 & 10.151383 & 23.180279 & 0.438 & 0.6615 & \\
\hline TypeNew & -42.717049 & 83.351375 & -0.512 & 0.6083 & \\
\hline Туретх & -4.979051 & 26.599889 & -0.187 & 0.8515 & \\
\hline $\begin{array}{l}\text { NumberFrequencies } \\
\text {--- }\end{array}$ & 0.010415 & 0.004557 & 2.286 & 0.0223 & $*$ \\
\hline Signif. codes: 0 ‘***, 0.00 & 01 ‘**’ 0.01 & ‘*’ 0.05 & 0.1 & , 1 & \\
\hline \multicolumn{6}{|c|}{$\begin{array}{l}\text { Residual standard error: } 79.92 \text { on } 3241 \text { degrees of freedom } \\
\text { Multiple R-squared: } 0.1073 \text {, Adjusted R-squared: } 0.1034 \\
\text { F-statistic: } 27.81 \text { on } 14 \text { and } 3241 \text { DF, p-value: }<2.2 \mathrm{e}-16\end{array}$} \\
\hline
\end{tabular}

Fig. 23: Multi-linear Regression Model Summary

The factors used in the aforementioned model were selected in accordance to the importance that they might have when applying for a given license. Nevertheless, we can also analyze these factors from a pure "data with no context" point of view. In this manner, we can implement a "Backward Variable selection" 34 to select the most suitable independent variables

\footnotetext{
${ }^{32}$ We used both the average frequency and the number of frequencies being requested.

${ }^{33}$ Only the independent variables Year, Duration and Type of License have statistical significance with $\operatorname{Pr}(>|t|)$ with codes 0 .

${ }^{34}$ Stepwise selection: Start with a model that includes all predictor variables, and then delete them one at a time until removing variables would not degrade the quality of the model.
}

for the model [14]. In our case the most significant factors are: Experimental Equipment, Calendar Year, License Type and Number of Frequencies ${ }^{35}$. However, even when using only these independent variables, it does not result in a valid multilinear prediction regression. The model would account for just $11 \%$ of the predictions with very low statistically significance.

The results of this more complete model, i.e., multi-linear regression, are not conclusive from a data mining point of view. The model has very low accountability with no statistical significance. Nonetheless, from an ERS regulation perspective, these results are very encouraging. They show that the outcome of a given application has almost no relationship with any requested, technical and non-technical, parameter. This is closely related to our results regarding the existing flexibility in the applications for experimental licenses.

\section{B. Determining the Most Relevant Parameters in the Applica- tion Process}

Any application presented for obtaining an experimental license is composed of several pieces of data, including technical and non-technical details. One of our goals in understanding what is behind the surface of the ERS licenses is to determine whether these pieces of information have some kind of "weighting hierarchy structure" associated to them ${ }^{36}$. In this light, we seek to construct an information model that help us determine the "importance" of these details when obtaining an experimental license. To fulfill this goal we utilize Decision Trees. This modeling tool "learns" the implied set of rules to classify an application as approved or denied given a set of data attributes. A tree is constructed with the most important factor in the top based on its own standing ${ }^{37}$ within the whole historical data [15].

The initial decision factor for our model was whether an application is approved or denied. However, since most of the dismissed applications are corrected ${ }^{38}$ and sent again to the FCC, there are very slight differences between granted and denied applications. Consequently, a decision tree using this factor was not viable at all. In light of this situation, we decided to use a more distinctive factor in our decision trees: the processing delay.

The resulting model for the period between 2007 and 2016 shows the year when the request was made as the most relevant parameter in any given application (Fig. 24). In fact, our decision tree shows that only in 2007 and 2008 other factors such as the duration of the license or if it was just a modification, have had an impact in the application delay.

\footnotetext{
${ }^{35}$ Variables selected through the "Backward Variable Selection" technique configured in stepwise selection mode.

${ }^{36}$ In other words, if any piece of information presented as part of an experimental application, has a greater influence in the outcome of the process that all other details.

${ }^{37}$ Calculated using the information gain, which is derived from the node and data entropy.

${ }^{38}$ Many changes required by the FCC correspond to additional paperwork or details to be presented within the application, rather than any substantial change in its core.
} 

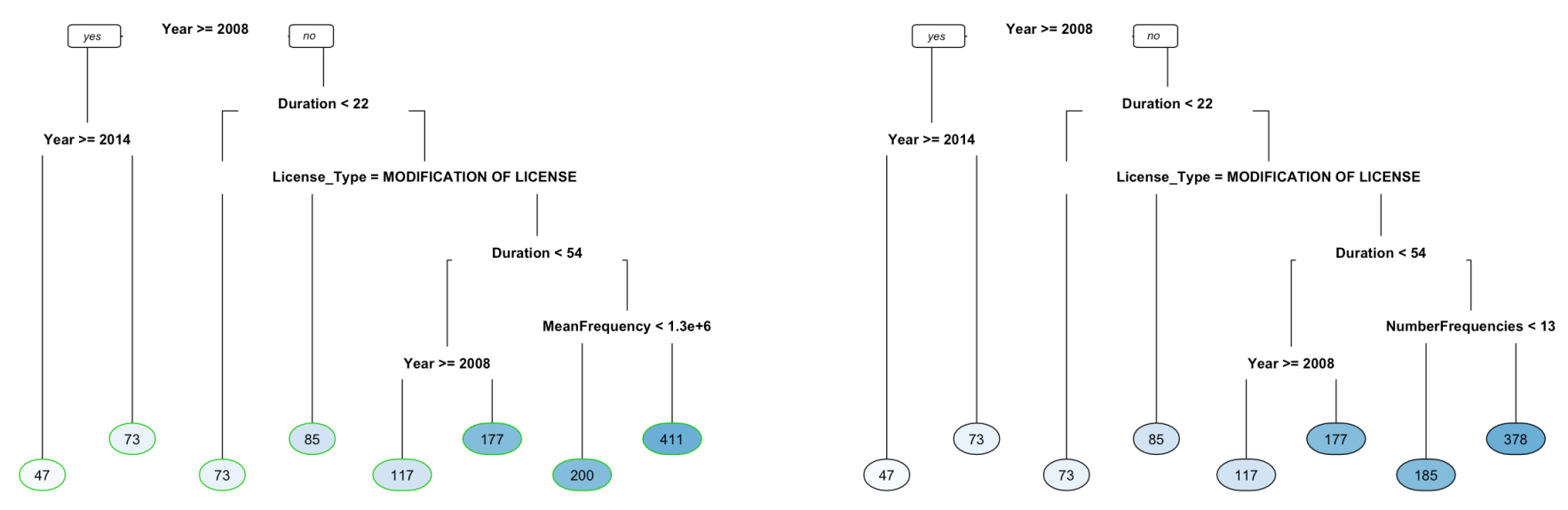

Fig. 24: Decision Trees for the Average Processing Delay (Left:Average Frequency, Right:Number of Frequencies)

In the case of core technical characteristics, frequency ${ }^{39}$ has a minimal impact, only affecting applications with a duration greater than 54 months. On the other hand, the influence of the mean power in the processing delay is, in fact, null.

The results obtained through decision tree modeling and the multi-linear techniques are very closely related. We can see that no other factors besides the year in which a request was received has had any impact on the outcome of an application during the past ten years. In fact, for both models, technical characteristics such as frequency and power have a minor or no impact at all. A possible explanation for these results could be traced back to the already shown feature of having no limitations in technical parameter choices within the ERS. Another plausible explanation is the utilization of the standardized electronic forms developed by the FCC, which have made the process of getting a license completely transparent for any requester.

\section{Relationship Among Applicants}

So far we have explored potential hidden trends and details about the requesting process of an experimental license, which is solely related to the FCC. Nonetheless, for a complete analysis, it is also necessary to seek possible similarities among the applicants who are obtaining authorizations. Based on the required classification of licensees, our final task, in this deeper exploration, is to find potential hidden relationships among the ERS licensees. We have selected clustering modeling as an appropriate tool for this exploration. The creation of groups allows us to organize similar data instances or objects into clusters ${ }^{40}$. Further, the proposed clustering technique, k-means, helps us to create a fixed number of groups (k) based on a distance function that finds the similarities between distinct applications [14].

The FCC categorizes the type of licensees into five fixed classes: Corporation, Association, Partnership, Individual and

\footnotetext{
${ }^{39} \mathrm{We}$ used both the average frequency and the number of frequencies being requested.

${ }^{40}$ Generally known as partitioning or segmentation.
}

Other. Consequently, we created a $\mathrm{k}=5$ clustering model to seek possible similarities among the members of this categorization scheme. The results of this approach are depicted in Fig. 25 and Table VI. In the same way as previous findings, we found that there is no significant difference among the applicants and their requested characteristics. Indeed, we can observe that all constructed clusters have at least a small percentage of each of the applicant types determined by the FCC. These results show the diversity of applicants in the different categories of the FCC's classification. Further, our findings exhibit that specific classes are not restricted to a particular applicant type. Indeed, license petitioners request different authorizations disregarding their assigned classification. Furthermore, as also depicted in Fig. 25, we observe that there is no overlapping between the constructed groups. Further, all members within the clusters are situated very close to each other ${ }^{41}$. These results show that the model is successfully differentiating among group members using all the pieces of information presented as part of their applications.

TABLE VI: Cluster Distribution based on the Applicant Type

\begin{tabular}{|c|r|r|r|r|r|}
\hline Cluster & Corp. & \multicolumn{1}{|c|}{ P'ship. } & Individual & \multicolumn{1}{c|}{ Assoc. } & \multicolumn{1}{c|}{ Other } \\
\hline $\mathbf{1}$ & $14.28 \%$ & $28.57 \%$ & $14.28 \%$ & $14.28 \%$ & $28.57 \%$ \\
\hline $\mathbf{2}$ & $25 \%$ & $25 \%$ & $12.5 \%$ & $12.5 \%$ & $25 \%$ \\
\hline $\mathbf{3}$ & $9.09 \%$ & $36.37 \%$ & $36.37 \%$ & $9.09 \%$ & $9.09 \%$ \\
\hline $\mathbf{4}$ & $21.05 \%$ & $10.52 \%$ & $15.78 \%$ & $31.57 \%$ & $21.05 \%$ \\
\hline $\mathbf{5}$ & $33.34 \%$ & $16.66 \%$ & $16.66 \%$ & $16.66 \%$ & $16.66 \%$ \\
\hline
\end{tabular}

This deeper data exploration have also shown some important results regarding the applicants and the application process for experimental licenses. First, we can see that applications for ERS authorizations are processed in a fairly manner ${ }^{42}$. Thus, it is shown that the processing time delay is not related to any factor besides the year in which the

\footnotetext{
${ }^{41}$ Only clusters 1 (Blue representation in Fig. 25) and 3 (Red representation in Fig. 25) present a single member that is not located close to the rest of the group members

${ }^{42}$ Our regression model was not able to predict any future processing delay when more details about the licenses were included
} 


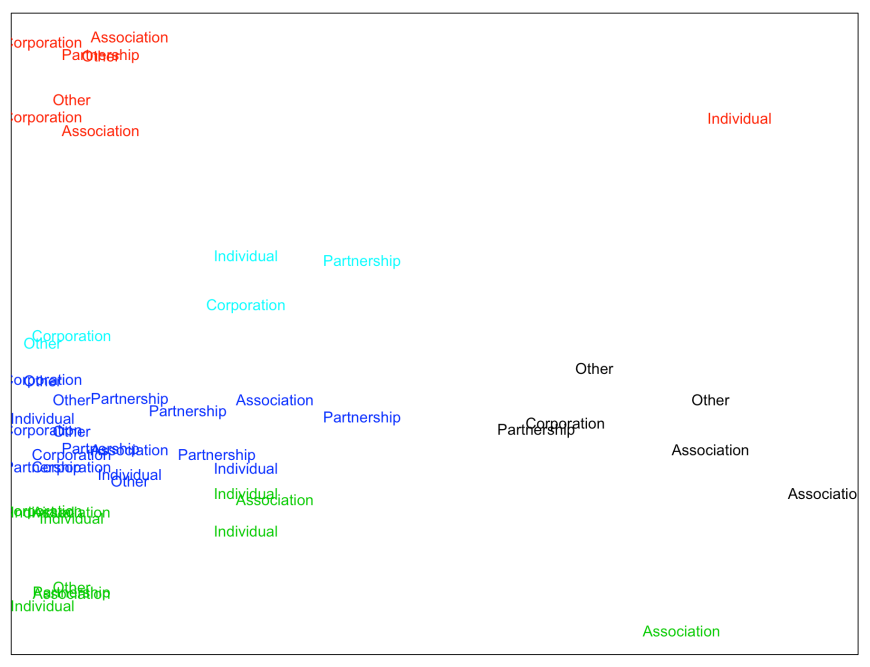

Fig. 25: Cluster Distribution based on the Applicant Type

request was presented. Furthermore, we saw that within the application process all the required and presented pieces of information have the same importance. Finally, when talking about applicants, it is shown that there is great diversity within the different FCC's categories. Thus, based solely on the obtained, technical and non-technical, parameters no group distinction can be made. These results imply that the process for obtaining a licenses is transparent from the applicant perspective and no parameter, technical or non-technical, is restricted to any particular type of licensee.

\section{EXPERIMENTAL LICENSES AND WIRELESS TECHNOLOGIES}

Experimental licenses are granted for a great variety of experiments. However, we wanted to conclude this work by directing our focus towards more specific applications. Hence, we analyze the utilization of experimental licenses for two well known areas within the wireless community: a proposed approach for spectrum sharing, TV White Spaces and, an emerging issue such as the development of $5 \mathrm{G}$ technologies.

\section{A. Spectrum Sharing: TV White Spaces (TVWS)}

Spectrum sharing and, in particular, the utilization of TV White Spaces is an interesting topic to cover in this analysis due to the constant concern of potential harmful interference with existing stations [3]. Since $2009^{43} 273$ licenses were issued by the FCC, 183 STAs and 90 conventional $^{44}$, for the exploration and experimentation of TVWS (See Fig. 26). It is worth pointing out the fact that the number of authorizations has considerably decreased since its peak between 2010 and 2013, where $79.12 \%$ of the licenses were granted.

To this point we have seen that most licenses mainly use non-experimental or commercial equipment as part of their authorizations. This is not the case for TVWS licenses. On

\footnotetext{
${ }^{43}$ No licenses are registered for TVWS in 2007 and 2008.

${ }^{44}$ Includes New, Modification and Renewals in the period.
}

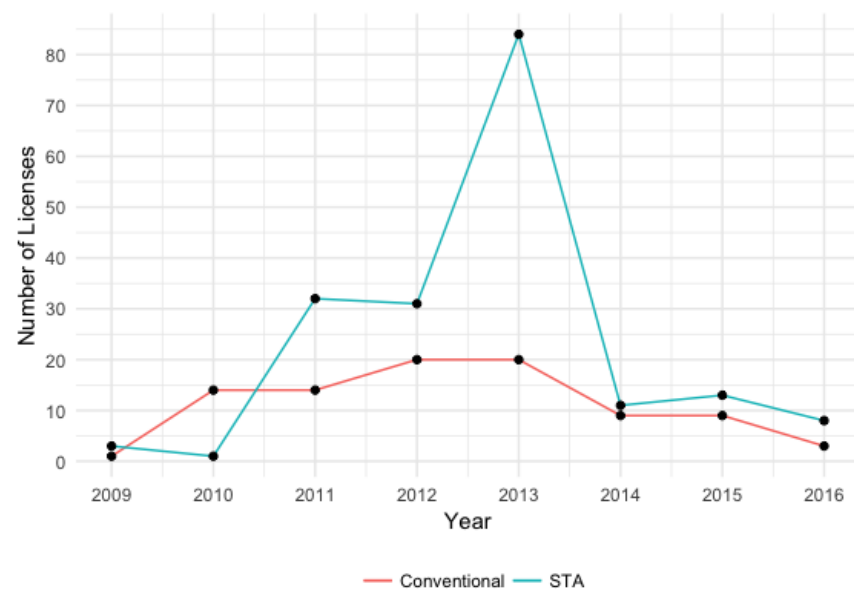

Fig. 26: TVWS: Number of Licenses

average, $62 \%$ of the total deployed equipment has been experimental since 2009 (Fig. 27). Moreover, in years such as 2003, 2009 and 2014 the exploration in TVWS was performed using only experimental equipment in the transmission stations.

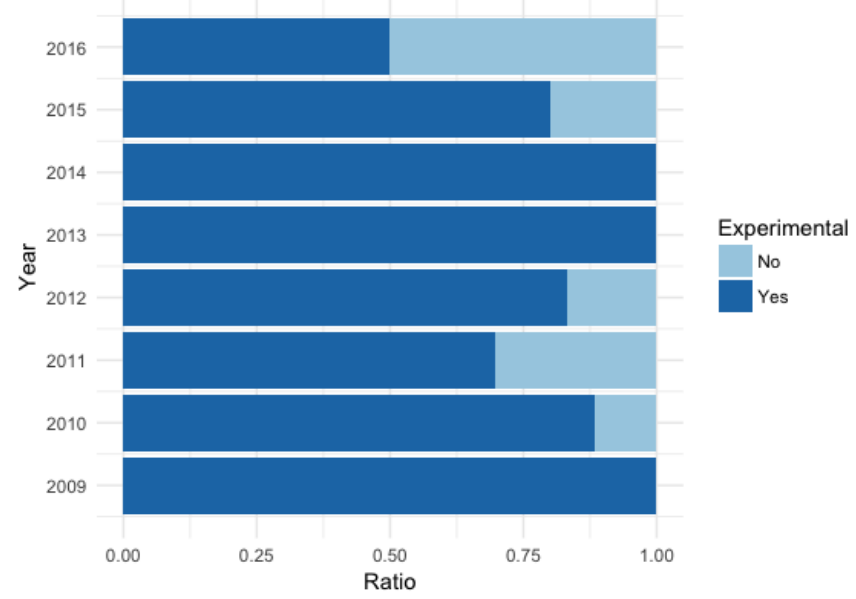

Fig. 27: TVWS: Experimental Equipment Distribution

The 270+ licenses issued for the development of TVWS utilized a total of 878 frequency assignations. These frequencies, as depicted in Fig. 28, are broadly distributed between the UHF and VHF television bands with a greater concentration towards higher UHF bands between 396 and $630 \mathrm{MHz}$.

An important factor when discussing potential spectrum sharing schemes such as TVWS is the allowable power. As expected, these authorizations use relatively low transmission power (See Fig. 29). Most experiments, including conventional and STA licenses, were only granted power levels of less than 4 watts, and $40 \%$ of licenses operate between 1 and 2 watts.

In this work we have explored frequency and power independently. Nevertheless, in the case of TVWS, we will explore how these two factors are related (Fig. 30). We observe that across all frequencies, the used transmission power is always 


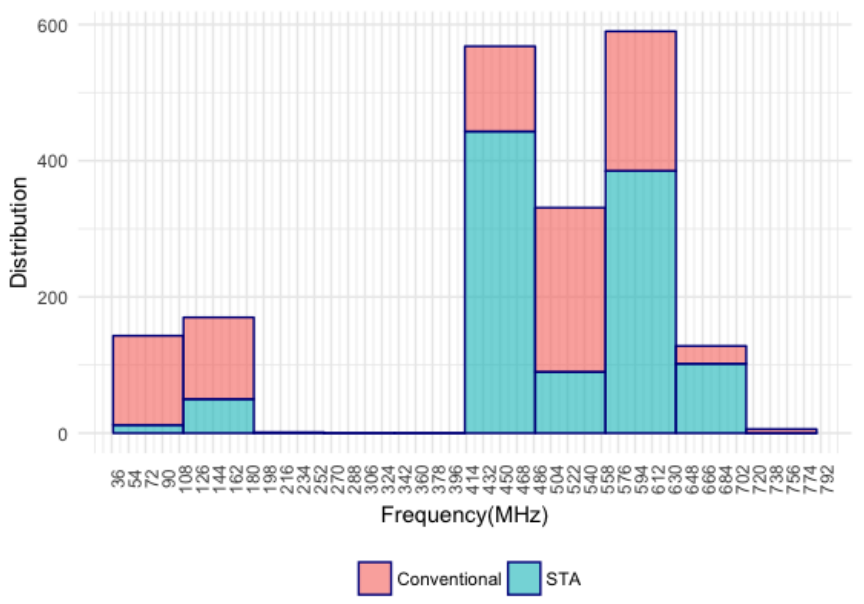

Fig. 28: TVWS: Frequency Distribution

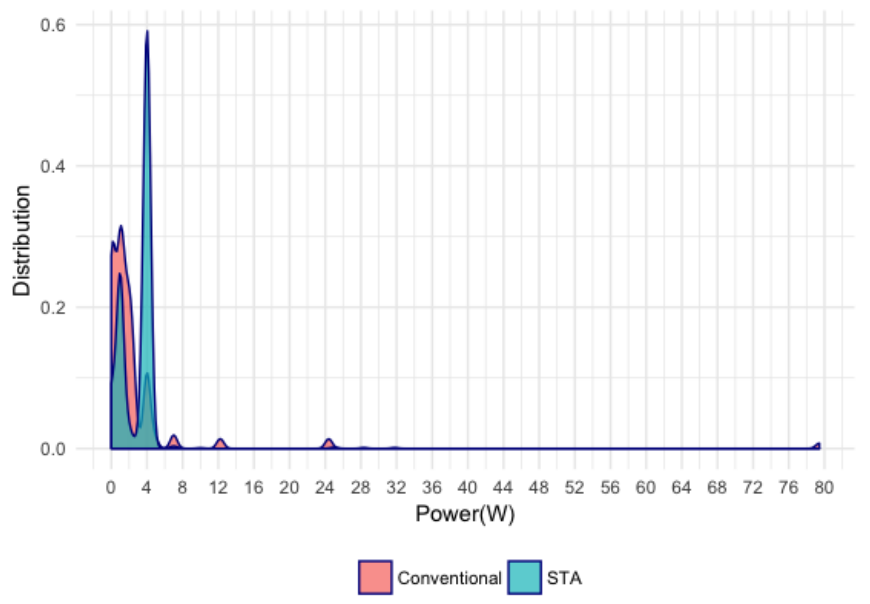

Fig. 29: TVWS: Power Distribution

low, less than $8 \mathrm{~W}$. In addition, only for channels from 7 to $11^{45}$ different power levels were actually tested, with the highest power being around $80 \mathrm{~W}$. We see that several experiments ${ }^{46}$ implement the same power-frequency set up.

Finally, to conclude our analysis of the relationship between the ERS and TVWS technologies, we explore the most common objectives behind obtaining a license in this area. First, note that any applicant can detail the "purpose of operation" of its experiment on an open text field ${ }^{47}$ within the application forms. Consequently, to actually obtain useful information about the purpose of operation behind TVWS, a text cleaning process and the construction of a term-document matrix were

\footnotetext{
${ }^{45}$ Frequencies: $174-204 \mathrm{MHz}$,

${ }^{46}$ Depicted as the size of the bubble in Fig. 30.

${ }^{47}$ No text limitations and no predefined options to choose from.
}

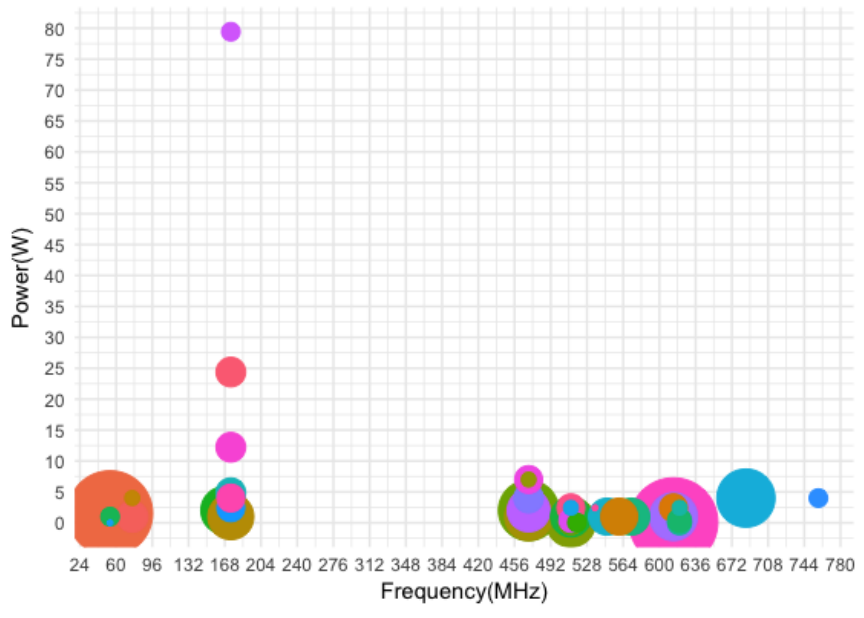

Fig. 30: TVWS: Assigned Frequency vs. Peak Power

necessary ${ }^{48}$ [2].

The goals behind TVWS authorizations and all other applications, shown in Section V-D3, are significantly different. These authorizations, as depicted in Fig. 31, are less focused on equipment testing or developing, and concentrated in other needs that are particular to the technology. We can see that the majority of licenses have the goal of bridging the spectrum allocation. Other less common uses of the licenses include the assessment of technical viability, providing contents and setting radios.

\section{serving region distinct
needs terrestrial challenges \\ apropriate spectrum allocato provide content distribution \\ spectrum bridge spectrum working spectrum bridge bridge spectrum allocation

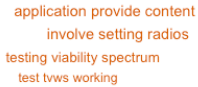

Fig. 31: TVWS: Most Common Purposes of Operation

\section{B. New Cellular Technologies: $5 G$}

It is undeniable that, at the moment, one of the most popular topics in the wireless community is the new cellular technology: 5G. This is also true for the ERS where over a 100 licenses have been granted for experimentation in this

\footnotetext{
${ }^{48}$ The same purpose of operation can be expressed in a wide range of ways. Consequently, the text cleaning process allow us to convert all these distinct expressions into similar terms. Once this is done, the most common terms are selected and mapped to each application through the term-document matrix. This allow us to find the most common goals in experimental licenses applications.
} 
area since $2010^{49}$. The number of applications has experienced a significant increase these past two years (Fig. 32) with the greatest number of licenses being granted in 2016 . We find that most licenses are granted as temporary authorizations rather than as conventional licenses.

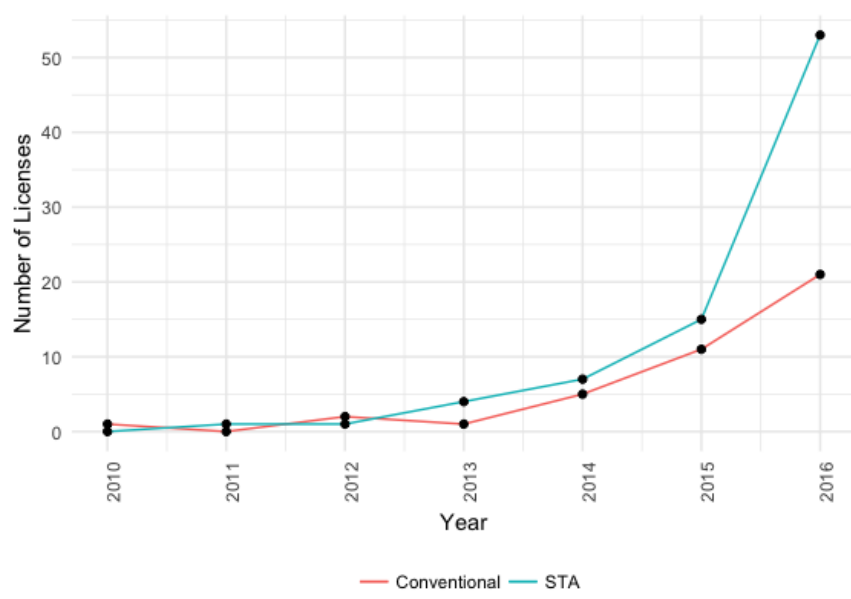

Fig. 32: 5G: Number of Licenses

In the analysis of TVWS we pointed out that, contrary to the majority of applications, most equipment being used is non-commercial. This trend is also evident in experimental licenses for the development of $5 \mathrm{G}$ technologies, where the average experimental equipment is even higher, approximately $82 \%$. Indeed, for years such as 2012 and 2015 the only instrumentation used in the ERS for 5G technologies was noncommercial (See Fig. 33).

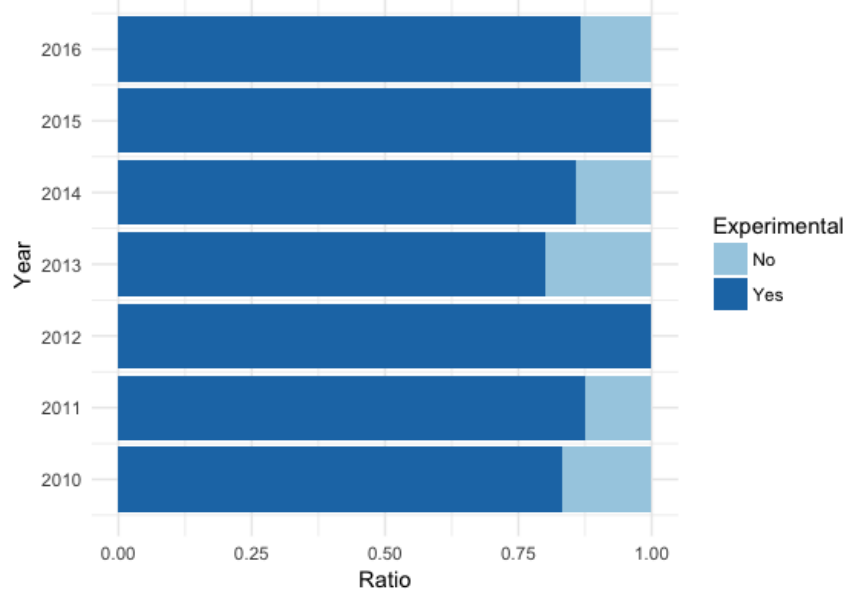

Fig. 33: 5G: Experimental Equipment Distribution

One of the most discussed and innovative details behind 5G technologies is its potential frequency allocation. As explored for example in [23] the main idea in the newest cellular technology is to explore bands over $6 \mathrm{GHz}$. In the case of ERS licenses, we observe this approach to be true.

\footnotetext{
${ }^{49}$ Before 2010 no licenses are registered for $5 \mathrm{G}$ related topics.
}

Most conventional licenses are being used in higher bands, between 20 and $45 \mathrm{GHz}$. This is also clear in the temporary authorizations distribution, which displays a particular peak located in the $28 \mathrm{GHz}$ band (Fig. 34). Further, we can point to applications for experimental licenses with authorizations in such bands: 0548-EX-PL-2010 and 0040-EX-ML-2012. These licenses were issued to the University of Texas at Austin to utilize 20 distributed frequencies with different power levels to test the theoretical coverage of 5G stations [21]. Nevertheless, this is not the only example of the relationship among high frequencies, the development of $5 \mathrm{G}$ technologies and experimental licenses. The FCC has already granted several STAs for equipment testing and development ${ }^{50}$.

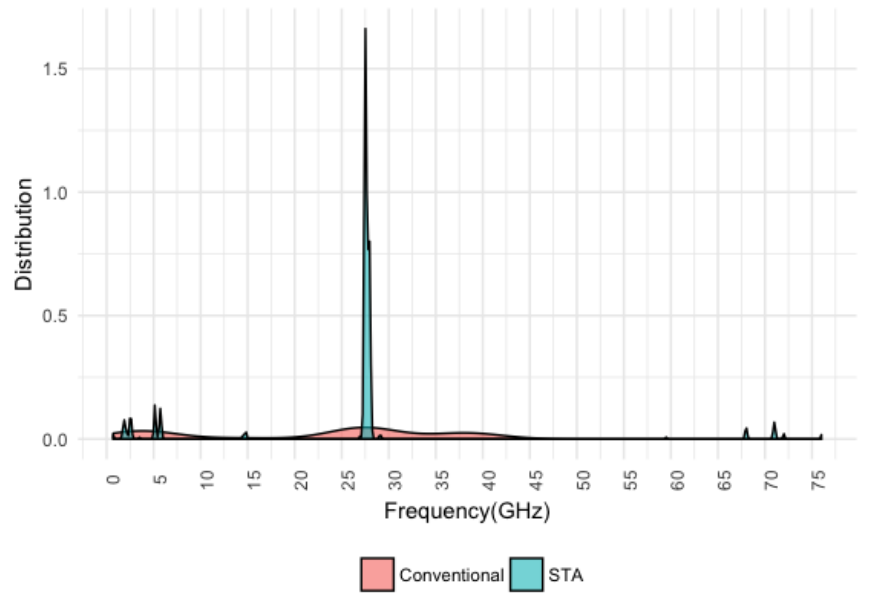

Fig. 34: 5G: Frequency Distribution

Another interesting detail to observe about $5 \mathrm{G}$ experimental authorizations is their power distribution. As depicted in Fig. 35 the power distribution is not the same for the special authorizations and conventional licenses. However, there are still some similarities worth mentioning. For instance, most of the transmission stations have been tested with low maximum power levels, i.e., less than $100 \mathrm{~W}$. In the case of STAs, the low power peak is comparable with the concentration on relatively high power levels around $600 \mathrm{~W}$. On the other hand, conventional licenses are concentrated under the $100 \mathrm{~W}$ threshold with some experimentation using higher power levels (200 and 600W).

As demonstrated in Fig. 36 by mapping frequency assignations to maximum allowable power in $5 \mathrm{G}$ experimental licenses we can observe a variety of power arrays being tested in different bands. Thus, for example in the $20 \mathrm{GHz}$ band we can see a concentration of experiments between 10 and 30 $\mathrm{W}$; however, all kinds of power assignations are also being tested. In addition, as we observe in Fig. 36 there is also a high concentration of experiments with low power levels, i.e., less than $5 \mathrm{~W}$, in the $5 \mathrm{GHz}$ range.

\footnotetext{
${ }^{50}$ For instance, the STA with Callsign WK9XII was assigned to AT\&T Services, for tests in the $27.5-28.5 \mathrm{GHz}$ band [4]
} 


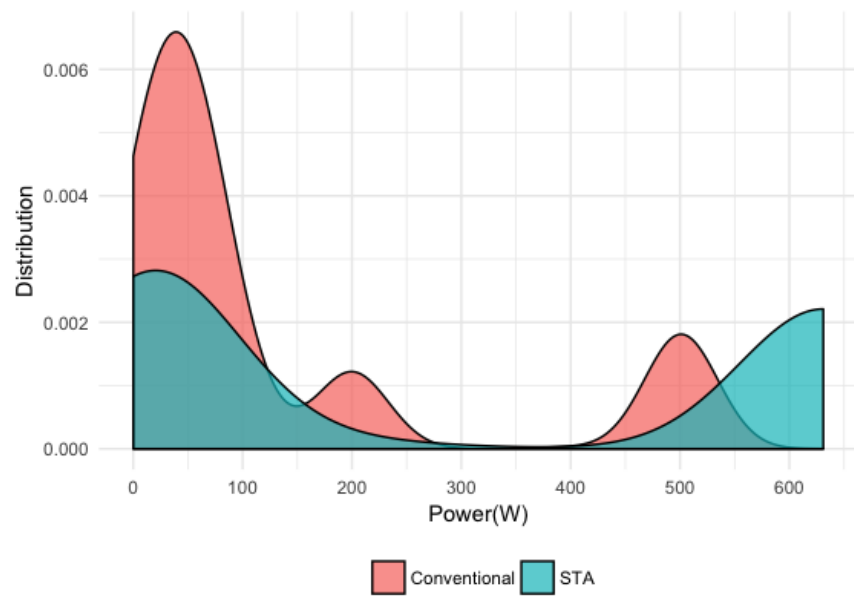

Fig. 35: 5G: Power Distribution

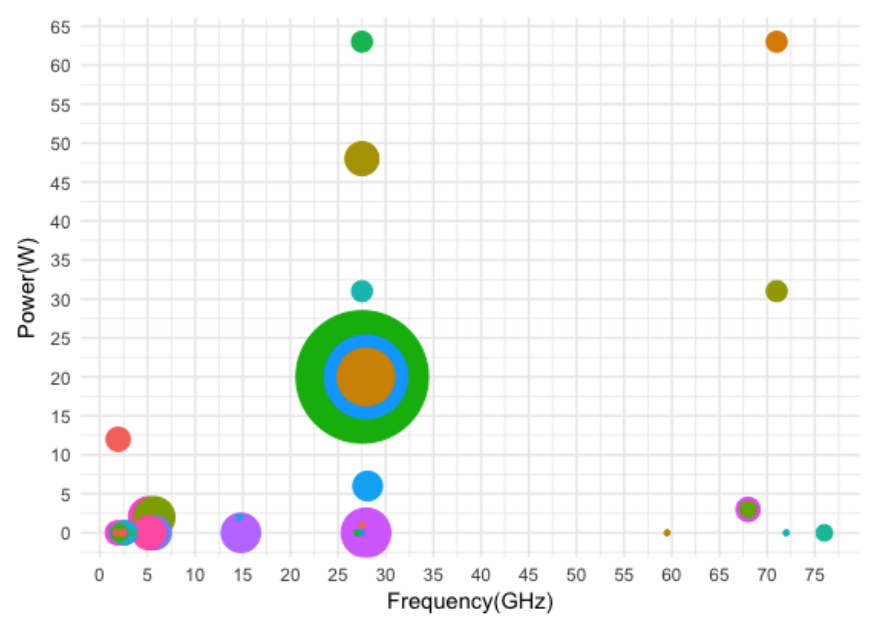

Fig. 36: 5G: Assigned Frequency vs. Peak Power

In Section V-D3 we explored the different ways in which STAs have been utilized. We pointed out that these licenses can be divided in four categories: Demonstrations, Developing, Analyze and Testing ${ }^{51}$. In the case of STAs granted for experimentation of $5 \mathrm{G}$ technologies the same distribution holds. The most common goal among these licenses is the demonstration of prototypes to customers (Fig. 37). However, this is not the only implementation of experimental licenses in the development of $5 \mathrm{G}$ technologies. For instance, other common purposes of operation include market trials and equipment testing.

This final section has allowed us to observe the relationship between experimental licenses and the development of wireless technologies. In this light, for example, we have seen the decrease in the number of authorizations for research in TVWS. Further, we observe the preferred frequencies and low power levels that were utilized in these set-ups. With regards to the development of $5 \mathrm{G}$, we saw the implementation of

\footnotetext{
${ }^{51}$ Classification for licenses not related to the coverage and support of televised events.
}

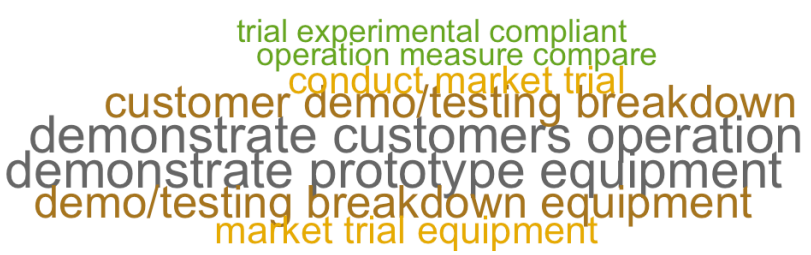

Fig. 37: Most Common Usages of 5G Licenses

transmission stations on high bands such as 28 and $38 \mathrm{GHz}$. Moreover, we can see the efforts to demonstrate and test the technology and its equipment as the main goals behind obtaining an experimental authorization in this area. These results highlight how innovation is allowed within the ERS authorization scheme. Further, different combinations of power and frequency have been authorized using mainly experimental equipment.

\section{CONCLUSIONS}

The analysis that we have performed has enabled us to explore the actual range of applicability and impact of the FCC's experimental licenses. Indeed, as a result of the amount of information that is publicly available, we have been able to construct a database that suits the purposes of our study.

Throughout this work, we have pointed out statistics on how different metrics have changed over the past ten years. For instance, we have observed how the number of assigned (granted) licenses has significantly increased during this period. On the other hand, the average delays associated with the licensing process have decreased from 100 to 23 days. These outcomes represent positive opportunities for those seeking access to experimental licenses.

We have also explored the great flexibility within the experimental licenses. Authorizations are being given for research in almost all frequency bands, with different maximum power levels and a variety of operation goals. Further, the deeper mining process of the scrapped information did not reveal any "hidden bias" in obtaining an experimental license or relationship between the type of licensee and the requested characteristics. Thus, we can see that the different technical and non-technical parts of an application are treated equally no matter what technical parameters, type of applicant, type of license are being requested.

In the exploration of the relationship between the development of wireless technologies and experimental licenses we found that it is actually in accordance with the objectives of innovation and access to spectrum of the ERS. Thus, most of the equipment being used is non-commercial or experimental. Moreover, the most common goals surrounding these experiments are a possible frequency allocation or prototype demonstrations for potential customers. 


\section{REFERENCES}

[1] Memorandum opinion and order and further notice of proposed rulemaking:. Promoting Expanded Opportunities for Radio Experimentation and Market Trials under Part 5 of the Commissions Rules and Streamlining Other Related Rules, 2015.

[2] Charu C Aggarwal and ChengXiang Zhai. Mining text data. Springer Science \& Business Media, 2012.

[3] David Borth, Randy Ekl, Bruce Oberlies, and Stu Overby. Considerations for successful cognitive radio systems in us tv white space. In New Frontiers in Dynamic Spectrum Access Networks, 2008. DySPAN 2008. 3rd IEEE Symposium on, pages 1-5. IEEE, 2008.

[4] Pedro Bustamante. Analysis of experimental licenses of the federal communications commission (fcc), January 2017.

[5] Ranveer Chandra, Thomas Moscibroda, Paramvir Bahl, Rohan Murty, George Nychis, and Xiaohui Wang. A campus-wide testbed over the tv white spaces. ACM SIGMOBILE Mobile Computing and Communications Review, 15(3):2-9, 2011.

[6] Shanzhi Chen and Jian Zhao. The requirements, challenges, and technologies for $5 \mathrm{~g}$ of terrestrial mobile telecommunication. IEEE Communications Magazine, 52(5):36-43, 2014.

[7] Federal Communications Commission. Conditions for use of outdoor test ranges for rf immunity testing. Technical report, 071996.

[8] Federal Communications Commission. Part 5: Experimental radio service. Code of Federal Regulations Title, 47, 2013.

[9] Federal Communications Commission. Promoting expanded opportunities for radio experimentation and market trials under part 5 of the commissions rules and streamlining other related rules. Report and Order FCC 13-15, 2013.

[10] Federal Communications Commission Spectrum Policy Task Force. Report of the unlicensed devices and experimental licenses working group, 2002.

[11] ITU-T. Nomenclature of the frequency and wavelength bands used in telecommunications. Technical report, 082015.

[12] Taeyoung Kim, Jeongho Park, Ji-Yun Seol, Suryong Jeong, Jaeweon Cho, and Wonil Roh. Tens of gbps support with mmwave beamforming systems for next generation communications. In 2013 IEEE Global Communications Conference (GLOBECOM), pages 3685-3690. IEEE, 2013.

[13] Julius Knapp. Open for business: Fcc's new experimental licensing system accepting new applications, 2017.

[14] Johannes Ledolter. Data mining and business analytics with R. John Wiley \& Sons, 2013.

[15] Bing Liu. Web data mining: exploring hyperlinks, contents, and usage data. Springer Science \& Business Media, 2007.

[16] George R MacCartney, Junhong Zhang, Shuai Nie, and Theodore S Rappaport. Path loss models for $5 \mathrm{~g}$ millimeter wave propagation channels in urban microcells. In 2013 IEEE Global Communications Conference (GLOBECOM), pages 3948-3953. IEEE, 2013.

[17] Shuai Nie, George R MacCartney, Shu Sun, and Theodore S Rappaport. $72 \mathrm{ghz}$ millimeter wave indoor measurements for wireless and backhaul communications. In PIMRC, pages 2429-2433, 2013.

[18] Office of Engineering and Technology. Experimental licensing system generic search, 2016.

[19] Federal Communications Commission Office of Engineering Technology. Federal Communications Commission (FCC) Experimental Licensing System (External) Users Manual. Federal Communications Commission, 445 12th Street SW, Washington, DC 20554, 3 edition, 2008.

[20] Inc Open Signal. The State of LTE (February 2016). Technical report, 022016.

[21] Theodore S Rappaport, Shu Sun, Rimma Mayzus, Hang Zhao, Yaniv Azar, Kevin Wang, George N Wong, Jocelyn K Schulz, Mathew Samimi, and Felix Gutierrez. Millimeter wave mobile communications for $5 \mathrm{~g}$ cellular: It will work! IEEE access, 1:335-349, 2013.

[22] Paul Struhsaker (TRIAS Research). 5g leaps forward, what you need to know about $5 \mathrm{~g}$ standards, spectrum and ecosystems, 2016.

[23] Wonil Roh, Ji-Yun Seol, Jeongho Park, Byunghwan Lee, Jaekon Lee, Yungsoo Kim, Jaeweon Cho, Kyungwhoon Cheun, and Farshid Aryanfar. Millimeter-wave beamforming as an enabling technology for 5g cellular communications: theoretical feasibility and prototype results. IEEE Communications Magazine, 52(2):106-113, 2014.

[24] Federal Communications Commission Rules. Regulations. Regulations, Experimental Radio Service, Part 5, 95, 2003.
[25] Peter Smulders. Exploiting the $60 \mathrm{ghz}$ band for local wireless multimedia access: Prospects and future directions. IEEE communications magazine, 40(1):140-147, 2002.

[26] Shu Sun and Theodore S Rappaport. Multi-beam antenna combining for $28 \mathrm{ghz}$ cellular link improvement in urban environments. In 2013 IEEE Global Communications Conference (GLOBECOM), pages 3754-3759. IEEE, 2013. 